\title{
Review Article \\ Singlet Fermion Assisted Dominant Seesaw with Lepton Flavor and Number Violations and Leptogenesis
}

\author{
M. K. Parida and Bidyut Prava Nayak \\ Centre of Excellence in Theoretical and Mathematical Sciences, SOA University, Khandagiri Square, Bhubaneswar 751030, India \\ Correspondence should be addressed to M. K. Parida; minaparida@soauniversity.ac.in
}

Received 23 July 2016; Revised 7 December 2016; Accepted 21 December 2016; Published 21 March 2017

Academic Editor: Alexey A. Petrov

Copyright (c) 2017 M. K. Parida and Bidyut Prava Nayak. This is an open access article distributed under the Creative Commons Attribution License, which permits unrestricted use, distribution, and reproduction in any medium, provided the original work is properly cited. The publication of this article was funded by SCOAP ${ }^{3}$.

\begin{abstract}
Embedding type I seesaw in GUTs, left-right gauge theories, or even in extensions of the SM requires large right-handed neutrino masses making the neutrino mass generation mechanism inaccessible for direct experimental tests. This has been circumvented by introducing additional textures or high degree of fine-tuning in the Dirac neutrino or right-handed neutrino mass matrices. In this work we review another new mechanism that renders type I seesaw vanishing but other seesaw mechanisms dominant. Such mechanisms include extended seesaw, type II, linear, or double seesaw. The linear seesaw, double seesaw, and extended seesaw are directly verifiable at $\mathrm{TeV}$ scale. New observable predictions for lepton flavor and lepton number violations by ongoing searches are noted. Type II embedding in $S O(10)$ also predicts these phenomena in addition to new mechanism for leptogenesis and displaced vertices mediated by gauge singlet fermions.
\end{abstract}

\section{Introduction}

Although the standard model (SM) of strong, weak, and electromagnetic interactions has enjoyed tremendous success through numerous experimental tests, it has outstanding failures in three notable areas: neutrino masses, baryon asymmetry of the universe, and dark matter. Two of the other conceptual and theoretical difficulties are that the SM cannot explain disparate values of its gauge couplings nor can it account for the monopoly of parity violation in weak interaction leaving out strong, electromagnetic, and gravitational interactions. In hitherto unverified extensions of the SM in the scalar, fermion, or gauge sectors, however, there are different theories for neutrino masses [1-11], which mainly exploit various seesaw mechanisms $[9,11-48]$.

The most popular method of neutrino mass generation has been through type I or canonical seesaw mechanism [1217] which was noted to apply in the simplest extension of the SM through right-handed ( $\mathrm{RH})$ neutrinos encompassing family mixings [17]. Most of the problems of the SM have potentially satisfactory solutions in the minimal left-right symmetric (LRS) [21, 49-52] grand unified theory based on $S O(10)$ [53-70]. Although the neutrino masses measured by the oscillation data [71-74] are most simply accommodated in $S O(10)$ if both the left-handed (LH) and the righthanded $(\mathrm{RH})$ neutrinos are Majorana fermions, alternative interpretations in favour of Dirac neutrino masses within the standard model paradigm have been also advanced $[11,75-78]$. Currently a number of experiments [79-84] on neutrinoless double beta $(0 \nu \beta \beta)$ decay are in progress to resolve the issue on the Dirac or Majorana nature of neutrinos [21-23].

Another set of physical processes under active experimental investigation are charged lepton flavor violating (LFV) decays, $\tau \rightarrow e \gamma, \tau \rightarrow \mu \gamma, \mu \rightarrow e \gamma$, and $\mu \rightarrow e \bar{e} e$, where the minimally extended SM embracing small neutrino masses and GIM mechanism predict branching ratios many orders smaller than their current experimental limits [8596]. However supersymmetric theories possess high potential to explain LFV decays closer to the current limits [97-104].

A special feature of left-right gauge theories [49-52] and $S O(10)$ grand unified theory (GUT) [53-60] is that 
the canonical seesaw formula [12-17] for Majorana neutrino masses is invariably accompanied by the type II seesaw formula [18-22]

$$
M_{\nu}=m_{\nu}^{\mathrm{II}}+m_{\nu}^{\mathrm{I}}=f v_{L}-M_{D} \frac{1}{M_{N}} M_{D}^{T} .
$$

The parameters entering into this hybrid seesaw formula have fundamentally appealing interpretations in Pati-Salam model $[49,50]$ or $S O(10)$ GUT. In $(1) M_{D}\left(M_{N}\right)$ is Dirac (RH Majorana) neutrino mass, $v_{L} \sim \lambda\left(v_{\mathrm{wk}}^{2} V_{R} / M_{\Delta_{L}}^{2}\right)$ is the induced vacuum expectation value (VEV) of the $\mathrm{LH}$ triplet $\Delta_{L}, V_{R}=S U(2)_{R} \times U(1)_{B-L}$ breaking VEV of the RH triplet $\Delta_{R}$, and $f$ is the Yukawa coupling of the triplets $\subset \overline{126}_{H}$ of $S O(10)$. The same Yukawa coupling $f$ also defines the RH neutrino mass $M_{N}=f V_{R}$. Normally, because of the underlying quark-lepton symmetry in $S O(10)$ or Pati-Salam model, $M_{D}$ is of the same order as $M_{u}$, the up quark mass matrix, that drives the canonical seesaw scale to be large, $M_{N} \sim 10^{14} \mathrm{GeV}$. In the LR theory based upon $S U(2)_{L} \times$ $S U(2)_{R} \times U(1)_{B-L} \times S U(3)_{C}\left(\equiv G_{2213}\right), M_{D} \sim M_{l}=$ charged lepton mass matrix. The neutrino oscillation data then pushes this seesaw scale to $M_{N} \sim 10^{10} \mathrm{GeV}$. Similarly the type II seesaw scale is also around this mass. With such high seesaw scales in nonsupersymmetric (non-SUSY) SO(10) model or LRS theory, there is no possibility of direct experimental verification of the seesaw mechanism or the associated $W_{R}$ boson mass in near future. Likewise, the predicted LFV decay rates are far below the experimental limits.

On the other hand, if experimental investigations at the Large Hadron Collider (LHC) [105, 106] are to confirm [107] TeV scale $W_{R}[9,108-111]$ or $Z_{R}$ boson production $[112,113]$ accompanied by RH Majorana neutrinos in the like-sign dilepton channel with jets, $p p \rightarrow l^{ \pm} l^{ \pm} j j X$, this should be also consistent with the neutrino oscillation data [71-74] through seesaw mechanisms. This is possible if the relevant seesaw scales are brought down to $\sim(1-10) \mathrm{TeV}$ (in the RH neutrino extension of the SM, explanation of neutrino oscillation data with baryon asymmetry of the universe has been addressed for $\mathcal{O}(1-10) \mathrm{GeV}$ type I seesaw scale [114-116]; interesting connections with double beta decay and dilepton production with displaced vertices at LHC have been also discussed with such low canonical seesaw scales [117] and the models need fine-tuning of the $\nu-N-\phi$ Yukawa coupling $y \sim 10^{-8}$ which is about 3 orders smaller than the electron Yukawa coupling; neutrino mass generation with standard model paradigm and their interesting applications have been discussed in a recent review [11]). Observable displaced vertices within SM extension have been also discussed in $[118,119]$ and references therein.

The scope and applications of type I seesaw to $\mathrm{TeV}$ scale $W_{R}$ boson models have been discussed in the recent interesting review [9]. In such models D-Parity is at first broken at high scale that makes the left-handed triplet much heavier than the $W_{R}$-mass but keeps $G_{2213}\left(g_{2 L} \neq g_{2 R}\right)$ unbroken down to much lower scale [55-57]. This causes the type II seesaw contribution of the hybrid seesaw formula of (1) to be severely damped out in the LHC scale $W_{R}$ models where type I seesaw dominates. But because $M_{N}$ is also at the TeV scale, the predicted type I seesaw contribution to light neutrino mass turns out to be $10^{6}-10^{11}$ times larger than the experimental values unless it is adequately suppressed while maintaining its dominance over type II seesaw. Such suppressions have been made possible in two ways: (i) using fine-tuned values of the Dirac neutrino mass matrices $M_{D}[9,117,120-122]$ and (ii) introducing specific textures to the fermion mass matrices $M_{D}$ and/or $M_{N}[98,108,123-136]$.

Possible presence of specific textures of constituent matrices in the context of inverse, linear, or type I seesaw models has been also explored [137-145]. More recently the hybrid seesaw ansatz for matter parity conserving $S O(10)$ has been applied to explain neutrino masses, dark matter, and baryon asymmetry of the universe without invoking any texture or intermediate scale in the nonsupersymmetric $S O(10)$ framework [146].

Even without going beyond the SM paradigm and treating the added $\mathrm{RH}$ neutrinos in type I seesaw as gauge singlet fermions at $\sim \mathrm{GeV}$ scale, rich structure of new physics has been predicted including neutrino masses, dark matter, and baryon asymmetry of the universe. The fine-tuned value of the associated Dirac neutrino Yukawa coupling in these models is $y \sim 10^{-7}$ [114-116].

There are physical situations where type II seesaw dominance, rather than type I seesaw or inverse seesaw, is desirable [17-23, 112, 147-162].

In the minimal case, being a mechanism driven by intermediate scale mass of LH triplet, type II seesaw may not be directly verifiable; nevertheless it can be clearly applicable to $\mathrm{TeV}$ scale $Z_{R}$ models in non-SUSY $S O(10)$ to account for neutrino masses [112] provided type I contribution is adequately suppressed. However, as in the fine-tuning of Dirac neutrino mass in the type I seesaw case in LR models, the induced VEV needed for type II seesaw can also be finetuned using more than one electroweak bidoublets reducing the triplet mass to lower scales accessible to accelerator tests. Looking to (1) and the structure of the induced VEV $v_{L}$, the most convenient method of suppressing type I seesaw with respect to type II seesaw is to make the type I seesaw scale $M_{N}=f V_{R}$ larger and the triplet mass much smaller, $M_{\Delta_{L}} \ll M_{N}$. This requires the $S U(2)_{R} \times U(1)_{B_{L}}$ breaking scale or $M_{W_{R}} \gg M_{\Delta_{L}}$. SUSY and non-SUSY SO(10) models have been constructed with this possibility and also in the case of split-SUSY $[157,160]$ where $M_{W_{R}} \simeq 10^{17} \mathrm{GeV}$. Obviously such models have no relevance in the context of TeV scale $W_{R}$ or $Z_{R}$ bosons accessible to LHC searches.

Whereas the pristine type I or type II seesaw are essentially high scale formulas inaccessible for direct verification and need fine-tuning or textures to bring them down to the $\mathrm{TeV}$ scale, the well known classic inverse seesaw mechanism [24] which has been also discussed by a number of authors [25-34] is essentially $\mathrm{TeV}$ scale seesaw. It has the high potential to be directly verifiable at accelerator energies and also by ongoing experiments on charged lepton flavor violations [85-96].

Even without taking recourse to string theories, in addition to the three RH neutrinos $\left(N_{i}, i=1,2,3\right)$, one more gauge singlet fermion per generation $\left(S_{i}, i=1,2,3\right)$ is added 
to the SM where the Lagrangian contains the $N-S$ mixing mass term $M$. The heavy Majorana mass term is absent for RH neutrinos which turn out to be heavy pseudo Dirac fermions. The introduction of the global lepton symmetry breaking term in the Lagrangian, $\mu_{S} S^{T} S$, gives rise to the well known classic inverse seesaw formula [24]

$$
m_{\nu}=\frac{M_{D}}{M} \mu_{S}\left(\frac{M_{D}}{M}\right)^{T} .
$$

Naturally small value of $\mu_{S}$ in the 't Hooft sense [163] brings down the inverse seesaw mechanism to the $\mathrm{TeV}$ scale without having the need to fine tune the associated Dirac mass matrix or Yukawa couplings. The presence of texture zeros in the constituent matrices of different types of seesaw formulas has been investigated consistently with neutrino oscillation data [138-140, 142, 143].

Recently models have been discussed using $\mathrm{TeV}$ scale heavy pseudo Dirac neutrinos $[109,164-168]$ where dominant RH Majorana mass term $M_{N} N N$ is either absent in the Lagrangian or negligible. In a contrasting situation [37, 38, 43], when $\mu_{S} \sim M_{\text {Planck }}$, the seesaw scale $M \sim M_{\mathrm{GUT}}$, and the model avoids the presence of any additional intermediate symmetry. While operating with the SM paradigm, it also dispenses with the larger Higgs representation $126_{H} \quad C$ $S O(10)$ in favour of much smaller one $16_{H}$ leading to the double seesaw. The heavy RH neutrinos with $M_{N} \sim 10^{13} \mathrm{GeV}$ turn out to be Majorana fermions instead of being pseudo Dirac. While this is an attractive scenario in SUSY SO(10) $[37,38]$, the coupling unification is challenging in the nonSUSY $S O(10)$.

As discussed above if type I seesaw is the neutrino mass mechanism at the $\mathrm{TeV}$ scale, it must be appropriately suppressed either by fine-tuning or by introducing textures to the relevant mass matrices [9]. On the other hand if type II seesaw dominance in LR models or $S O(10)$ is to account for neutrino masses, $W_{R}, Z_{R}$ boson masses must be at the GUT-Planck scale in the prevailing dominance mechanisms $[157,160]$.

In view of this, it would be quite interesting to explore, especially in the context of nonsupersymmetric $S O(10)$, possible new physics implications when the would-be dominant type I seesaw cancels out exactly and analytically from the light neutrino mass matrix even without needing any finetuning or fermion mass textures in $M_{D}$ and/or $M_{N}$. The complete cancellation of type I seesaw in the presence of heavy RH Majorana mass term $M_{N} N N$ was explicitly proved in $[45,46]$ in the context of SM extension when both $N_{i}$ and $S_{i}$ are present manifesting in heavy $\mathrm{RH}$ neutrinos and lighter singlet fermions. We call this as gauge singlet fermion assisted extended seesaw dominance mechanism. Since then the mechanism has been utilised in explaining baryon asymmetry of the universe via low scale leptogenesis $[45,46]$ and the phenomenon of dark matter (DM) [169] along with cosmic ray anomalies [170]. More recently this extended seesaw mechanism for neutrino masses in the $\mathrm{SM}$ extension has been exploited to explain the $\mathrm{keV}$ singlet fermion DM along with low scale leptogenesis [171].
In the context of LR intermediate scales in SUSY SO(10), this mechanism has been applied to study coupling unification and leptogenesis [172-174] under gravitino constraint. Application to non-SUSY LR theory originating from PatiSalam model [175] and non-SUSY $S O(10)$ with TeV scale $W_{R}$, $Z_{R}$ bosons have been made $[176,177]$ with the predictions of a number of experimentally testable physical phenomena by low-energy experiments and including the observed dilepton excess at LHC [110]. In these models the singlet fermion assisted type I seesaw cancellation mechanism operates and the extended seesaw (or inverse seesaw) formula dominates.

Following the standard lore in type II seesaw dominant models, the dominant double beta decay rate in the $W_{L}-W_{L}$ channel is expected to be dominated by the exchange of the LH Higgs triplet $\Delta_{L}$ carrying $|B-L|=2$. As such the predicted decay rate tends to be negligible in the limit of larger Higgs triplet mass. But it has been shown quite recently [112] that the type II seesaw dominance can occur assisted by the gauge singlet fermion but with a phenomenal difference. Even for large LH triplet mass in such models that controls the type II seesaw formula for light neutrino masses and mixings, the double beta decay rate in the $W_{L}-W_{L}$ channel remains dominant as it is controlled by the light singlet fermion exchanges. Other attractive predictions are observable LFV decays, nonunitarity effects, and resonant leptogenesis mediated by $\mathrm{TeV}$ scale quasi-degenerate singlet fermions of the second and third generations. The model has been noted to have its origin in non-SUSY $S O(10)$ [112]. All the three types of gauge singlet fermions in these models mentioned above are Majorana fermions on which we focus in this review.

This article is organised in the following manner. In Section 2 we explain how the Kang-Kim mechanism [45, 46] operates within the SM paradigm extended by singlet fermions. In Section 3 we show how a generalised neutral fermion mass matrix exists in the appropriate extensions of the SM, LR theory, or $S O(10)$. In Section 4 we show emergence of the other dominant seesaw mechanism including the extended or inverse seesaw and type II seesaw and cancellation of type I seesaw. Predictions for LFV, CP violation, and nonunitarity effect are discussed in Section 5. Predictions on double beta decay mediated by light singlet fermions in the $W_{L}-W_{L}$ channel are discussed in Section 6 where we have given its mass limits from the existing experimental data. Applications to resonant leptogenesis mediated by $\mathrm{TeV}$ scale singlet fermions in MSSM and SUSY $S O(10)$ are briefly discussed in Section 7. Singlet fermion assisted leptogenesis in non-SUSY $S O(10)$ is discussed in Section 8. This work is summarized in Section 9 with conclusions.

\section{Mechanism of Extended Seesaw Dominance in SM Extension}

Using the explicit derivation of Kang and Kim [45, 46], here we discuss how the type I contribution completely cancels out paving the way for the dominance of extended seesaw mechanism. The SM is extended by introducing $\mathrm{RH}$ neutrinos $N_{i}(i=1,2,3)$ and an additional set of fermion 
singlets $S_{i}(i=1,2,3)$, one for each generation. After electroweak symmetry breaking, the Yukawa Lagrangian in the charged lepton mass basis gives for the neutral fermions

$$
\begin{aligned}
\mathscr{L}_{\text {mass }}= & \left(M_{D} \bar{\nu} N+\frac{1}{2} M_{N} N^{T} N+M \bar{N} S+\text { h.c }\right) \\
& +\mu_{S} S^{T} S
\end{aligned}
$$

where $M_{D}$ is the Dirac neutrino mass matrix which is equal to $Y\langle\phi\rangle, Y$ being the Yukawa matrix. This gives the $9 \times 9$ neutral fermion mass matrix on the $\left(\nu, N^{c}, S\right)$ basis as follows:

$$
\mathscr{M}_{v}=\left(\begin{array}{ccc}
0 & M_{D} & 0 \\
M_{D}^{T} & M_{N} & M^{T} \\
0 & M & \mu_{S}
\end{array}\right) .
$$

The type I seesaw cancellation leading to dominance of extended seesaw (or inverse seesaw) [24] proceeds in two steps: as $M_{N} \gg M \gg M_{D}, \mu_{S}$, it is legitimate to integrate out the RH $N_{i}$ fields at first leading to the corresponding effective Lagrangian

$$
\begin{aligned}
-\mathscr{L}_{\mathrm{eff}}= & \left(M_{D} \frac{1}{M_{N}} M_{D}^{T}\right)_{\alpha \beta} \nu_{\alpha}^{T} v_{\beta} \\
& +\left(M_{L}+M_{D} \frac{1}{M_{N}} M^{T}\right)_{\alpha m}\left(\overline{\nu_{\alpha}} S_{m}+\overline{S_{m}} \nu_{\alpha}\right) \\
& +\left(M \frac{1}{M_{N}} M^{T}\right)_{m n} S_{m}^{T} S_{n}+\mu_{S} S^{T} S .
\end{aligned}
$$

Then diagonalisation of the $9 \times 9$ neutral fermion mass matrix including the result of $\mathscr{L}_{\text {eff }}$ gives conventional type I seesaw term and another of opposite sign leading to the cancellation. The light neutrino mass predicted is the same as in the inverse seesaw case given in (2).

It must be emphasized that the earlier realisations of the classic inverse seesaw formula [24] were possible [25-32, 34] with vanishing $\mathrm{RH}$ Majorana mass $M_{N}=0$ in (12).

Under the similar condition in which the type I seesaw cancels out the Majorana mass $m_{S}$ of the sterile neutrino and its mixing angle $\theta_{S}$ with light neutrinos are governed by

$$
\begin{aligned}
m_{S} & =\mu_{S}-M \frac{1}{M_{N}} M^{T} \sim-M \frac{1}{M_{N}} M^{T}, \\
\tan 2 \theta_{S} & =2 \frac{M_{D}}{M} .
\end{aligned}
$$

As $\mu_{S}$ is naturally small, it is clear that type I seesaw now controls the gauge singlet fermion mass, although it has no role to play in determining the $\mathrm{LH}$ neutrino mass. These results have been shown to emerge $[47,109,110,112,176,177]$ from $S O(10)$ with gauge fermion singlet extensions by following the explicit block diagonalisation procedure in two steps while safeguarding the hierarchy $M_{N} \gg M>M_{D}$, $\mu_{S}$ with the supplementary condition $\mu_{S} M_{N}<M^{2}$.

\section{Generalised Neutral Fermion Mass Matrix}

A left-right symmetric (LRS) gauge theory $G_{2213 D}\left(g_{2 L}=g_{2 R}\right)$ at higher scale $\left(\mu=M_{P}\right)$ is known to lead to $\mathrm{TeV}$ scale asymmetric LR gauge theory $G_{2213}\left(g_{2 L} \neq g_{2 R}\right)$ via D-Parity breaking [55-57]. This symmetry further breaks to the SM gauge symmetry by the VEV of the RH triplet $\Delta_{R}(1,3,-2,1)$ leading to massive $W_{R}, Z_{R}$ bosons and $\mathrm{RH}$ neutrinos at the intermediate scale $M_{R}$. Instead of $G_{2213 D}\left(g_{2 L}=g_{2 R}\right)$ it is possible to start directly from $S O(10)$ which has been discussed at length in a number of investigations that normally leads to the type I $\oplus$ type II hybrid seesaw formula. In the absence of additional sterile neutrinos, the neutral fermion matrix is standard $6 \times 6$ form. Here we discuss how a generalised $9 \times 9$ neutral fermion mass matrix that emerges in the presence of additional singlet fermions contains the rudiments of various seesaw formulas. As noted in Section 1, the derivation of the minimal classic inverse seesaw mechanism [24] has been possible in theories with gauge singlet fermion extensions of the SM [25-34, 178]. Extensive applications of this mechanism have been discussed and reported in a number of recent reviews [11, 99-102, 179, 180]. Exploring possible effects on invisible Higgs decays [181-183], prediction of observable lepton flavor violation as a hall mark of the minimal classic inverse seesaw mechanism has attracted considerable attention earlier and during recent investigations [103, 184-189]. The effects of massive gauge singlet fermions have been found to be consistent with electroweak precision observables [190192]. Earlier its impact on a class of left-right symmetric models has been examined [193-195]. Prospects of lepton flavor violation in the context of linear seesaw and dynamical left-right symmetric model have been also investigated earlier [178].

It is well known that 15 fermions of one generation plus a right-handed neutrino form the spinorial representation 16 of $S O(10)$ grand unified theory $[53,54]$. In addition to three generation of fermions $16_{i}(i=1,2,3)$, we also include one $S O(10)$-singlet fermion per generation $S_{i}(i=1,2,3)$. We note that such singlets under the LR gauge group or the SM can originate from the nonstandard fermion representations in $S O(10)$ such as $45_{F}$ or $210_{F}$.

Under $G_{2213}$ symmetry the fermion and Higgs representations are as follows:

\section{Fermions}

$$
\begin{aligned}
Q_{L} & =\left(\begin{array}{l}
u \\
d
\end{array}\right)_{L}\left(2,1, \frac{1}{3}, 3\right), \\
Q_{R} & =\left(\begin{array}{l}
u \\
d
\end{array}\right)_{R}\left(1,2,-\frac{1}{3}, 3^{*}\right), \\
L & =\left(\begin{array}{c}
v_{l} \\
l
\end{array}\right)_{L}(2,1,-1,1), \\
R & =\left(\begin{array}{c}
N_{l} \\
l
\end{array}\right)_{R}(1,2,-1,1), \\
S_{i} & =(1,1,-1,1) .
\end{aligned}
$$


Higgs

$$
\begin{gathered}
\phi=\left(\begin{array}{ll}
\phi_{1}^{0} & \phi_{2}^{+} \\
\phi_{1}^{-} & \phi_{2}^{0}
\end{array}\right)(2,2,0,1) \\
\Delta_{L}=\left(\begin{array}{cc}
\frac{\Delta_{L}^{+}}{\sqrt{2}} & \Delta_{L}^{++} \\
\Delta_{L}^{0} & -\frac{\Delta_{L}^{+}}{\sqrt{2}}
\end{array}\right)(3,1,-2,1) \\
\Delta_{R}=\left(\begin{array}{cc}
\frac{\Delta_{R}^{+}}{\sqrt{2}} & \Delta_{R}^{++} \\
\Delta_{R}^{0} & -\frac{\Delta_{R}^{+}}{\sqrt{2}}
\end{array}\right)(1,3,-2,1) \\
\chi_{L}=\left(\begin{array}{l}
\chi_{L}^{0} \\
\chi_{L}^{-}
\end{array}\right)(2,1,-1,1) \\
\chi_{R}=\left(\begin{array}{l}
\chi_{R}^{0} \\
\chi_{R}^{-}
\end{array}\right)(1,2,-1,1) \\
\eta_{o}(1,1,0,1)
\end{gathered}
$$

where $\eta_{o}$ is a D-Parity odd singlet with transformation property $\eta_{o} \rightarrow-\eta_{o}$ under $L \rightarrow R$. When this singlet acquires VEV $\left\langle\eta_{o}\right\rangle \sim M_{P}$, D-Parity breaks along with the underlying left-right discrete symmetry but the asymmetric LR gauge theory $G_{2213}$ is left unbroken down to the lower scales. The $G_{2213}$ gauge theory can further break down to the SM directly by the VEV of RH Higgs triplet $\Delta_{R}(1,3,-2,1) \subset$ $126_{H} \subset S O(10)$ or the RH Higgs doublet $\chi_{R}(1,2,-1,1) \subset$ $16_{H} \subset S O(10)$. The D-Parity odd (even) singlets $\eta_{o}\left(\eta_{e}\right)$ were found to occur naturally in $S O(10)$ GUT theory [55-57]. Designating the quantum numbers of submultiplets under Pati-Salam symmetry $S U(2)_{L} \times S U(2)_{R} \times S U(4)_{C}\left(\equiv G_{224}\right)$, the submultiplet $(1,1,1) \subset 210_{H} \subset S O(10)$ is $\eta_{o}$ whereas the submultiplet $(1,1,1) \subset 54_{H} \subset S O(10)$ is $\eta_{e}$. Likewise the neutral component of the submultiplet $(1,1,15) \subset 45_{H} \subset$ $S O(10)$ behaves as $\eta_{o}$, but that in $(1,1,15) \subset 210_{H}$ behaves as $\eta_{e}$. Thus the GUT scale symmetry breaking $S O(10) \rightarrow G_{224 D}$ can occur by the VEV of $54_{H}$ in the direction $\left\langle\eta_{e}\right\rangle \sim M_{\mathrm{GUT}}$, but $S O(10) \rightarrow G_{224}$ can occur by the VEV of $210_{H}$ in the direction $\left\langle\eta_{o}\right\rangle \sim M_{\mathrm{GUT}}$. Likewise $S O(10) \rightarrow G_{2213 D}$ can occur by the VEV of the neutral component $(1,1,0,1)_{H} \subset$ $(1,1,15)_{H} \subset 210_{H}$, but $S O(10) \rightarrow G_{2213}$ can occur by the VEV of the neutral component of $(1,1,0,1)_{H} \subset(1,1,15)_{H} \subset$ $45_{H}$. As an example, one minimal chain with TeV scale LR gauge theory proposed recently in the context of like-sign dilepton signals observed at LHC is

$$
\begin{aligned}
S O(10) \stackrel{\left(M_{U}=M_{P}\right)}{\longrightarrow} & S U(2)_{L} \times S U(2)_{R} \times U(1)_{B-L} \\
& \times S U(3)_{C}\left[G_{2213}\right] \\
& \stackrel{\left(M_{R}\right)}{\longrightarrow} S U(2)_{L} \times U(1)_{Y} \times S U(3)_{C}[S M] \\
& \stackrel{\left(M_{Z}\right)}{\longrightarrow} S U(3)_{C} \times U(1)_{Q} .
\end{aligned}
$$

In this symmetry breaking pattern all LH triplets and doublets is near the GUT scale, but RH triplets or doublets are near the $G_{2213}$ breaking intermediate scale $M_{R}$ which could be $\sim($ few-100) TeV. Out of two minimal models with GUT scale D-Parity breaking satisfying the desired decoupling criteria $M_{N} \gg M \gg M_{D}, \mu_{S}[110,112]$, dominance of extended seesaw in the presence of gauge singlet fermions has been possible in [110] with single $G_{2213}$ intermediate scale corresponding to TeV scale $W_{R}$ and $Z_{R}$ bosons. The extended seesaw dominance in the presence of fermion singlets in $S O(10)$ has been also realised including additional intermediate symmetries $G_{2214 D}$ and $G_{224}$ where observable proton decay, TeV scale $Z_{R}$ boson and RH Majorana neutrinos, observable proton decay, $n-\bar{n}$ oscillation, and rare kaon decay have been predicted. Interestingly the masses of $W_{R}$ boson and leptoquark gauge bosons of $S U(4)_{C}$ have been predicted at $\sim 100 \mathrm{TeV}$ which could be accessible to planned LHC at those energies where $W_{R}$ boson scale $\sim(100-1000) \mathrm{TeV}$ matching with observable $n-\bar{n}$ oscillation and rare kaon decay have been predicted. But the heavy $\mathrm{RH}$ neutrino and $Z_{R}$ boson scales being near $\mathrm{TeV}$ scale have been predicted to be accessible to LHC and planned accelerators $[176,177]$. That non-SUSY GUTs with two-intermediate scales permit a low mass $Z_{R}$ boson was noted much earlier [196].

In (9), instead of breaking directly to SM, the $G_{2213}$ breaking may occur in two steps $G_{2213} \rightarrow G_{2113}$ SM where $G_{2113}$ represents the gauge symmetry $S U(2)_{L} \times U(1)_{R} \times$ $U(1)_{B-L} \times S U(3)_{C}\left[G_{2113}\right]$. This promises the interesting possibility of TeV scale $Z_{R}$ boson with the constraint $M_{W_{R}} \gg$ $M_{Z_{R}}$. Thus the model can be discriminated from the direct LR models if $Z_{R}$ boson is detected at lower mass scales than the $W_{R}$-boson. There are currently ongoing accelerator searches for this extra heavy neutral gauge boson. This has been implemented recently with type II seesaw dominance in the presence of added fermion singlets [112]. As we will discuss below both these types of models predict light neutrinos capable of mediating double beta decay rates in the $W_{L}-W_{L}$ channel saturating the current experimental limits. In addition resonant leptogenesis mediated by heavy sterile neutrinos has been realised in the model of [112].

The $G_{2213}$ symmetric Yukawa Lagrangian descending from $S O(10)$ symmetry can be written as

$$
\begin{aligned}
\mathscr{L}_{\text {Yuk }}= & \sum_{i=1,2} Y_{i}^{\ell} \bar{\psi}_{L} \psi_{R} \Phi_{i}+f\left(\psi_{R}^{c} \psi_{R} \Delta_{R}+\psi_{L}^{c} \psi_{L} \Delta_{L}\right) \\
& +y_{\chi}\left(\bar{\psi}_{R} S \chi_{R}+\bar{\psi}_{L} S \chi_{L}\right)+\text { h.c., }
\end{aligned}
$$

where $\Phi_{1,2} \subset 10_{H_{1}, H_{2}}$ are two bidoublets, $\left(\Delta_{L}, \Delta_{R}\right) \subset 126_{F}$ and $\left(\chi_{L}, \chi_{R}\right) \subset 16_{H}$.

Including the induced VEV contribution to $\Delta_{L}$, the Yukawa mass term can be written as

$$
\begin{aligned}
& \mathscr{L}_{\text {mass }} \\
& =\left(M_{D} \bar{\nu} N+\frac{1}{2} M_{N} N^{T} N+M \bar{N} S+M_{L} \overline{\nu_{L}} S+\text { h.c }\right) \\
& \quad+m_{\nu}^{\mathrm{II}} \nu^{T} v+\mu_{S} S^{T} S .
\end{aligned}
$$

Here the last term denotes the gauge invariant singlet mass term where naturalness criteria demand $\mu_{S}$ to be a very small 
parameter. In the $\left(\nu, S, N^{C}\right)$ basis the generalised form of the $9 \times 9$ neutral fermion mass matrix after electroweak symmetry breaking can be written as

$$
\mathscr{M}_{v}=\left(\begin{array}{ccc}
m_{v}^{\mathrm{II}} & M_{L} & M_{D} \\
M_{L}^{T} & \mu_{S} & M^{T} \\
M_{D}^{T} & M & M_{N}
\end{array}\right),
$$

where $M_{D}=Y\langle\Phi\rangle, M_{N}=f v_{R}, M=y_{\chi}\left\langle\chi_{R}^{0}\right\rangle$, and $M_{L}=$ $y_{\chi}\left\langle\chi_{L}^{0}\right\rangle$. In this model the symmetry braking mechanism and the VEVs are such that $M_{N}>M \gg M_{D}$. The LH triplet scalar mass $M_{\Delta_{L}}$ and RH neutrino masses being at the heaviest mass scales in the Lagrangian, this triplet scalar field and the $\mathrm{RH}$ neutrinos are at first integrated out leading to the effective Lagrangian at lower scales $[45,46,112]$ as follows:

$$
\begin{aligned}
-\mathscr{L}_{\mathrm{eff}}= & \left(m_{\nu}^{\mathrm{II}}+M_{D} \frac{1}{M_{N}} M_{D}^{T}\right)_{\alpha \beta} v_{\alpha}^{T} \nu_{\beta} \\
& +\left(M_{L}+M_{D} \frac{1}{M_{N}} M^{T}\right)_{\alpha m}\left(\overline{\nu_{\alpha}} S_{m}+\overline{S_{m}} \nu_{\alpha}\right) \\
& +\left(M \frac{1}{M_{N}} M^{T}\right)_{m n} S_{m}^{T} S_{n}+\mu_{S} S^{T} S .
\end{aligned}
$$

\section{Cancellation of Type I Seesaw and Dominance of Others}

4.1. Cancellation of Type I Seesaw. Whereas the heaviest $\mathrm{RH}$ neutrino mass matrix $M_{N}$ separates out trivially, the other two $3 \times 3$ mass matrices $\mathscr{M}_{v}$, and $\mathscr{M}_{S}$ are extracted through various steps of block diagonalisation. The details of various steps are given in [112, 176, 177].

$$
\begin{aligned}
\mathscr{M}_{v}= & m_{v}^{\mathrm{II}}+\left(M_{D} M_{N}^{-1} M_{D}^{T}\right)-\left(M_{D} M_{N}^{-1} M_{D}^{T}\right) \\
& +M_{L}\left(M^{T} M_{N}^{-1} M\right)^{-1} M_{L}^{T} \\
& -M_{L}\left(M^{T} M_{N}^{-1} M\right)^{-1}\left(M^{T} M_{N}^{-1} M_{D}^{T}\right) \\
& -\left(M_{D} M_{N}^{-1} M\right)\left(M^{T} M_{N}^{-1} M\right)^{-1} M_{L}^{T} \\
& +M_{D} M^{-1} \mu_{S} M_{D} M^{-1}{ }^{T} \\
\mathscr{M}_{\mathcal{S}}= & \mu_{S}-M_{N}^{-1} M^{T}+\cdots, \\
\mathscr{M}_{\mathcal{N}}= & M_{N} .
\end{aligned}
$$

From the first of the above three equations, it is clear that the type I seesaw term cancels out with another of opposite sign resulting from block diagonalisation. Then the generalised form of the light neutrino mass matrix turns out to be

$$
\begin{aligned}
\mathscr{M}_{v}= & f v_{L}+M_{L} M^{-1} M_{N}\left(M^{T}\right)^{-1} M_{L}^{T} \\
& -\left[M_{L} M^{-1} M_{D}^{T}+M_{D}\left(M_{L} M^{-1}\right)^{T}\right] \\
& +\frac{M_{D}}{M} \mu_{S}\left(\frac{M_{D}}{M}\right)^{T} .
\end{aligned}
$$

In different limiting cases this generalised light neutrino mass matrix reduces to the corresponding well known neutrino mass formulas.

4.2. Linear Seesaw and Double Seesaw. With $M_{L}=y_{\chi} v_{\chi_{L}}$ that induces $\nu$-S mixing, the second term in (15) is the double seesaw formula

$$
\mathscr{M}_{v}^{\text {(double) }}=M_{L} M^{-1} M_{N}\left(M^{T}\right)^{-1} M_{L}^{T}
$$

The third term in (15) represents the linear seesaw formula

$$
\mathscr{M}_{v}^{(\text {linear })}=-\left[M_{L} M^{-1} M_{D}^{T}+M_{D}\left(M_{L} M^{-1}\right)^{T}\right] .
$$

Similar formulas have been shown to emerge from single-step breaking of SUSY GUT models $[39,40,44]$ which require the presence of three gauge singlet fermions.

Using the D-Parity breaking mechanism of $[55,56]$, an interesting model of linear seesaw mechanism in the context of supersymmetric $S O(10)$ with successful gauge coupling unification [197] has been suggested in the presence of three gauge singlet fermions. A special feature of this linear seesaw, compared to others $[39,40,44]$, is that the neutrino mass formula is suppressed by the SUSY GUT scale but it is decoupled from the low $U(1)_{B-L}$ breaking scale. In addition to prediction of $\mathrm{TeV}$ scale superpartners, the model provides another important testing ground through manifestation of extra $Z^{\prime}$ boson at LHC or via low-energy neutrino scattering experiment [198].

4.3. Type II Seesaw. When VEV $\left\langle\chi_{L}\right\rangle=0$ or becomes negligible and $\mu_{S}=0$ in (2), type II seesaw dominates leading to

$$
m_{v} \simeq f v_{L}
$$

As noted briefly in Section 1, in the conventional models [157, 160] of type II seesaw dominance in $S O(10)$, the $W_{R}, Z_{R}$ boson masses have to be at the GUT-Planck scale. As a phenomenal development, this singlet fermion assisted type II seesaw dominance permits $U(1)_{B-L}$ breaking scale associated with $G_{2213}$ or $G_{2113}$ breaking (i.e., the $W_{R}$ and $Z_{R}$ boson masses) accessible to accelerator energies including LHC. At the same time the heavy $N-S$ mixing mass terms $M_{i}(i=1,2,3)$ at the $\mathrm{TeV}$ scale are capable of mediating observable LFV decay rates closer to their current experimental values [85-96] as discussed in Section 5. Consequences of this new type II seesaw dominance with $\mathrm{TeV}$ scale $Z_{R}$ boson mass have been investigated in detail [112] in which charged triplet mediated LFV decay rates are negligible but singlet fermion decay rates are observable. Also predictions of observable double beta decay rates close to their experimental limits are discussed below in Section 6. While the principle of such a dominance is clearly elucidated in this derivation, the details of the model with $\mathrm{TeV}$ scale $G_{2213}$ symmetry will be reported elsewhere.

4.4. Extended Seesaw. It is quite clear that the classic inverse seesaw formula [24] of (2) for light neutrino mass emerges 
when the LH triplet mass is large and the VEV $\left\langle\chi_{L}\right\rangle=0$ which is possible in a large class of non-SUSY models with left-right, Pati-Salam, and $S O(10)$ gauge groups with $D$-Parity broken at high scales [55-57] with $M_{N}=0$ leading to RH neutrinos as heavy pseudo Dirac fermions. Particularly in SO(10) some non-SUSY examples are $[109,166]$ and SUSY examples are $[164,165,199]$. The mechanism operates without supersymmetry provided we reconcile with gauge hierarchy problem and some non-SUSY models are [109, 110, 175, 176].

As noted in Section 1, the derivation of classic inverse seesaw mechanism [24-34] has $M_{N}=0$ in (12). More recent applications in LRS and GUTs have been discussed with relevant reference to earlier works in [126, 166, 167, 200-204].

In this section we have discussed that, in spite of the presence of the heavy Majorana mass term of RH neutrino, each of the three seesaw mechanisms, (i) extended seesaw, (ii) Type II seesaw, and (iii) linear seesaw or double seesaw, can dominate as light neutrino mass ansatz when the respective limiting conditions are satisfied. Also the seesaw can operate in the presence of $\mathrm{TeV}$ scale $G_{2213}$ or $G_{2113}$ gauge symmetry originating from non-SUSY $S O(10)[112,176,177]$. As the $\mathrm{TeV}$ scale theory spontaneously breaks to low-energy theory $U(1)_{e m} \times S U(3)_{C}$ through the electroweak symmetry breaking of the standard model, these seesaw mechanisms are valid in the SM extensions with suitable Higgs scalars and three generations of $N_{i}$ and $S_{i}$. For example without taking recourse to LR gauge theory type II seesaw can be embedded into the SM extension by inclusion of LH Higgs triplet $\Delta_{L}(3,-2,1)$ with $Y=-2$. The induced VEV can be generated by the trilinear term $\lambda M_{\mathrm{tr}} \cdot \Delta_{L}^{\dagger} \phi^{\dagger} \phi^{\dagger}[205,206]$. The origin of such induced VEV in the direct breaking of $S O(10) \rightarrow$ SM is well known.

4.5. Hybrid Seesaw. In the minimal $S O(10)$, without extra fermion singlets, one example of hybrid seesaw with type I $\oplus$ type II is given in (1). There are a number of investigations where this hybrid seesaw has been successful in parametrising small neutrino masses with large mixing angles along with $\theta_{13} \sim 8^{\circ}$ in SUSY SO(10) [148-153] and LR models. But the present mechanism of type I seesaw cancellation suggests a possible new hybrid seesaw formula as a combination of type $\mathrm{II} \oplus$ Linear $\oplus$ Extended seesaw as revealed from (15). Neutrino physics phenomenology may yield interesting new results with this new combination with additional degrees of freedom to deal with neutrino oscillation data and leptogenesis covering coupling unification in $S O(10)$ which has a very rich structure for dark matter.

Using the D-Parity breaking mechanism of $[55,56]$, an interesting model of linear seesaw mechanism in the context of supersymmetric $S O(10)$ with successful gauge coupling unification [197] has been suggested in the presence of three gauge singlet fermions. A special feature of this linear seesaw, compared to others [39,40], is that the neutrino mass formula is suppressed by the SUSY GUT scale but decoupled from the low $U(1)_{B-L}$ breaking scale which can be even at $\sim$ few $\mathrm{TeV}$. This serves as a testing ground through manifestation of extra $Z^{\prime}$ boson at LHC or via low-energy neutrino scattering experiments [198]. Being a SUSY model it also predicts TeV scale superpartners expected to be visible at LHC.
4.6. Common Mass Formula for Sterile Neutrinos. In spite of different types of seesaw formulas in the corresponding limiting cases the formula for sterile neutrino mass remains the same as in (6) which does not emerge from the classic inverse seesaw approach with $M_{N}=0$.

We conclude this section by noting that the classic inverse seesaw mechanism was gauged at the $\mathrm{TeV}$ scale through its embedding in non-SUSY $S O(10)$ with the prediction of experimentally accessible $Z^{\prime}$ boson, LFV decays, and nonunitarity effects [166]. The possibility of gauged and extended inverse seesaw mechanism with dominant contributions to both lepton flavor and lepton number nonconservation was at first noted in the context Pati-Salam model in [175] and in the context of non-SUSY $S O(10)$ in $[176,177]$ with type I seesaw cancellation. The generalised form of hybrid seesaw of (15) in non-SUSY SO(10) with type I cancellation was realised in [112]. As a special case of this model, the experimentally verifiable phenomena like extra $Z^{\prime}$ boson, resonant leptogenesis, LFV decays, and double beta decay rates closer to the current search limits were decoupled from the intermediate scale type II seesaw dominated neutrino mass generation mechanism. Proton lifetime prediction for $p \rightarrow$ $e+\pi^{0}$ mode also turns out to be within the accessible range.

\section{Predictions for LFV Decays, CP Violation, and Nonunitarity}

The presence of nonvanishing neutrino masses with generational mixing evidenced from the oscillation data [71-74], in principle, induces charged lepton flavor violating (LFV) decays. For a recent review see [99] and references therein. The observed neutrino mixings through weak charged currents lead to nonconservation of lepton flavor numbers $L_{e}$, $L_{\mu}$, and $L_{\tau}$ resulting in the predictions of $\mu \rightarrow e+\gamma, \tau \rightarrow e+\gamma$, $\tau \rightarrow \mu+\gamma, \mu \rightarrow e e \bar{e}$, and a host of others [85-96]. If the nonSUSY SM is minimally extended to embrace tiny neutrino masses and mixings through GIM mechanism as the only underlying source of charged lepton flavor violation, the loop mediated branching ratio is

$$
\operatorname{Br}\left(\ell_{\alpha} \longrightarrow \ell_{\beta}+\gamma\right) \simeq \frac{\alpha}{32 \pi}\left|\sum_{j=2,3} U_{\alpha i} U_{\beta j}^{*} \frac{\Delta m_{j 1}^{2}}{M_{W}^{2}}\right|^{2} .
$$

These branching ratios turn out to be $\leq 10^{-53}$ ruling out any possibility of experimental observation of the decay rates. In high intermediate scale non-SUSY $S O(10)$ models with Dirac neutrino mass matrix $M_{D}$ similar to the up quark mass matrix $M_{u}$, the type I seesaw ansatz for neutrino masses constrain the heavy RH neutrino masses $M_{N} \geq 10^{12} \mathrm{GeV}$ resulting in branching ratio values $\leq 10^{-40}$ which are far below the current experimental limits. Another drawback of the model is that the underlying neutrino mass generation mechanism and the predicted $W_{R}$ boson mass cannot be verified directly.

On the other hand SUSY GUTs are well known to provide profound predictions of CP-violations and LFV decay branching ratios closer to the current experimental limits in spite of their high scale seesaw mechanisms for neutrino masses. Some of the extensively available reviews on this 
subject are $[97,99,100,203,207]$. The superpartner masses near $100-1000 \mathrm{GeV}$ are necessary for such predictions.

As profound applications of the classic inverse seesaw mechanism it has been noted that the presence of heavy pseudo Dirac fermions would manifest through LFV decays $[33,184-188]$ and also in lepton number violation $[208,209]$. They are also likely to contribute to the modifications of the electroweak observables [190-192] keeping them within their allowed limits. It has been also emphasized that the SUSY inverse seesaw mechanism for neutrino masses further enhances the LFV decay rates [184]. As a direct test of the seesaw mechanism, these heavy particles with masses near the $\mathrm{TeV}$ scale can be produced at high energy colliders including LHC $[109,110,112,164,165,167]$. Other interesting signatures have been reviewed in [11].

More interestingly a linear seesaw formula has been predicted from supersymmetric $S O(10)$ with an extra $Z^{\prime}$ boson mass accessible to $\mathrm{LHC}$ [42]. The TeV scale classic inverse seesaw mechanism and $W_{R}$ gauge boson masses have been embedded in SUSY SO(10) with rich structure for leptonic $\mathrm{CP}$ violation, nonunitarity effects, and LFV decay branching ratios accessible to ongoing experiments [164, 165]. The impact of such a model with TeV scale pseudo Dirac type RH neutrinos has been investigated on proton lifetime predictions and leptogenesis [199].

As supersymmetry has not been experimentally observed so far, an interesting conceptual and practical issue is to confront LFV decay rates accessible to ongoing experimental searches along with the observed tiny values of light neutrino masses. In this section we summarise how, in the absence of SUSY, the classic inverse seesaw and the extended seesaw could still serve as powerful mechanisms to confront neutrino mass, observable lepton flavor violation $[109,166]$, and, in addition, dominant lepton number violation [110, 175-177] in non-SUSY SO(10). The possibilities of detecting TeV scale $W_{R}$ bosons have been also explored recently in non-SUSY $S O(10)[110,111,175-177]$. Besides earlier works in left-rightsymmetric model [210] and those cited in Sections 1-4, more recent works include $[61-66,78,211-217]$ and references cited in these papers.

In contrast to negligible LFV decay rates and branching ratios predicted in the non-SUSY SM modified by GIM mechanism, we discuss in Section 5.2 how the non-SUSY $S O(10)$ predicts the branching ratios in the range $10^{-13}-10^{-16}$ consistent with small neutrino masses dictated by classic inverse seesaw, extended inverse seesaw, or type II seesaw in the presence of added fermion singlets.

5.1. Neutrino Mixing and Nonunitarity Matrix. The light neutrino flavor state is now a mixture of three mass eigenstates $\widehat{v}_{i}$, $\widehat{S}_{i}$, and $\widehat{N}_{i}$ as follows:

$$
v_{\alpha}=U_{\alpha, i} \widehat{v}_{i}+\mathscr{V}_{\alpha, i} \widehat{S}_{i}+\mathcal{N}_{\alpha, i} \widehat{N}_{i}
$$

where in the diagonal bases of $S_{i}$ and $N_{i} \mathscr{V}_{\alpha, i}=\left(M_{D} / M\right)_{\alpha, i}$ and $\mathcal{N}_{\alpha, i}=\left(M_{D} / M_{N}\right)_{\alpha, i}$. In cases where the matrices $M$ and $M_{N}$ are nondiagonal, the corresponding flavor mixing matrices are taken as additional factors to define $\nu-S$ or $\nu-N$ mixing matrices. For the sake of simplicity, treating the $N-S$ mixing mass matrix $M$ as diagonal,

$$
M=\operatorname{diag}\left(M_{1}, M_{2}, M_{3}\right),
$$

and, under the assumed hierarchy $M_{N} \gg M$, the formula for the nonunitarity deviation matrix element $\eta_{\alpha \beta}$ has been defined in the respective cases [110, 112, 175-177, 218-231] as follows:

$$
\begin{aligned}
\eta & =\frac{1}{2} X \cdot X^{\dagger}=M_{D} M_{R}^{-2} M_{D}^{\dagger}, \\
\eta_{\alpha \beta} & =\frac{1}{2} \sum_{k=1,2,3} \frac{M_{D_{\alpha k}} M_{D_{\beta k}}^{*}}{M_{k}^{2}} .
\end{aligned}
$$

The Dirac neutrino mass matrix $M_{D}$ needed for the fit to neutrino oscillation data through extended seesaw formula and prediction of nonunitarity effects has been derived from the GUT scale fit of charged fermion masses in the case of non-SUSY SO(10) $[110,112,166,176,177]$. For this purpose, the available data at the electroweak scale on charged fermion masses and mixings are extrapolated to the GUT scale [232]. The fitting is done following the method of [233] by suitably adding additional contributions due to VEVs of additional bidoublets or higher dimensional operators, wherever necessary. In the inverse seesaw case with almost degenerate heavy pseudo Dirac neutrinos, $M_{D}$ has been derived in the case of SUSY $S O(10)$ with $\mathrm{TeV}$ scale $G_{2213}$ symmetry $[164,165]$ and in non-SUSY $S O(10)$ with $\mathrm{TeV}$ scale $G_{2113}$ symmetry [166]. In the case of extended seesaw dominance in non-SUSY $S O(10)$ it has been derived in [110, 175-177] whereas for type II seesaw dominance it has been derived in [112]. The value of $M_{D}$ thus derived at the GUT scale is extrapolated to the $\mathrm{TeV}$ scale following the top-down approach. It turns out that such values are approximately equal to the one shown in the following section in (32).

For the general nondegenerate case of the $M$ matrix, ignoring the heavier $\mathrm{RH}$ neutrino contributions and saturating the upper bound $\left|\eta_{\tau \tau}\right|<2.7 \times 10^{-3}$ gives

$$
\eta_{\tau \tau}=\frac{1}{2}\left[\frac{0.1026}{M_{1}^{2}}+\frac{7.0756}{M_{2}^{2}}+\frac{6762.4}{M_{3}^{2}}\right]=2.7 \times 10^{-3} .
$$

By inspection this equation gives the lower bounds $M_{1}>$ $4.35(\mathrm{GeV}), M_{2}>36.2(\mathrm{GeV})$, and $M_{3}>1120(\mathrm{GeV})$. And for the degenerate case $M_{\text {Deg }}=1213 \mathrm{GeV} . M(1213,1213$, 1212) GeV. For the partial degenerate case of $M_{1}=M_{2} \neq M_{3}$ the solutions can be similarly derived as in $[112,176]$ and one example is $M(100,100,1319.67) \mathrm{GeV}$.

Experimentally constrained lower bounds of the nonunitarity matrix elements are

$$
\begin{aligned}
& \left|\eta_{\tau \tau}\right| \leq 2.7 \times 10^{-3}, \\
& \left|\eta_{\mu \mu}\right| \leq 8.0 \times 10^{-4}, \\
& \left|\eta_{e e}\right| \leq 2.0 \times 10^{-3} \\
& \left|\eta_{e \mu}\right| \leq 3.5 \times 10^{-5}, \\
& \left|\eta_{e \tau}\right| \leq 8.0 \times 10^{-3} \\
& \left|\eta_{\mu \tau}\right| \leq 5.1 \times 10^{-3}
\end{aligned}
$$


TABLE 1: Predictions of moduli and phases of nonunitarity matrix $\eta_{\alpha \beta}$ as a function of allowed values of masses $M_{1}, M_{2}$, and $M_{3}$. The Dirac neutrino matrix is the same as in [112].

\begin{tabular}{|c|c|c|c|c|c|c|c|}
\hline$m_{R_{1}}=m_{R_{2}}(\mathrm{GeV})$ & $m_{R_{3}}(\mathrm{GeV})$ & $\left|\eta_{e \mu}\right|$ & $\delta_{e \mu}$ & $\left|\eta_{e \tau}\right|$ & $\delta_{e \tau}$ & $\left|\eta_{\mu \tau}\right|$ & $\delta_{\mu \tau}$ \\
\hline 1213.11 & 1213.11 & $2.737 \times 10^{-8}$ & 1.920 & $4.543 \times 10^{-7}$ & 1.78 & $2.318 \times 10^{-5}$ & $2.391 \times 10^{-7}$ \\
\hline 500 & 1280 & $8.132 \times 10^{-7}$ & 1.326 & $3.746 \times 10^{-7}$ & 1.456 & $2.426 \times 10^{-5}$ & $2.723 \times 10^{-5}$ \\
\hline 100 & 1119.67 & $6.543 \times 10^{-6}$ & 0.728 & $4.834 \times 10^{-6}$ & 0.974 & $2.975 \times 10^{-5}$ & $8.932 \times 10^{-4}$ \\
\hline 50 & 1545.31 & $7.652 \times 10^{-6}$ & 0.203 & $9.754 \times 10^{-6}$ & 0.342 & $3.424 \times 10^{-5}$ & $2.813 \times 10^{-3}$ \\
\hline
\end{tabular}

Out of several estimations of the elements of $\eta$-matrix [110, $112,166,175-177]$ carried out in non-SUSY $S O(10)$, here we give one example of [112]. Using the Dirac neutrino mass matrix from [112] and allowed solutions of $M_{i}$, the values of the $\eta_{\alpha \beta}$ parameters and their phases as functions of $M_{i}$ are determined using (22). These results are presented in Table 1.

5.2. LFV Decay Branching Ratios versus Neutrino Mass. The most important outcome of nonunitarity effect is expected to manifest through ongoing experimental searches for LFV decays such as $\tau \rightarrow e \gamma, \tau \rightarrow \mu \gamma$, and $\mu \rightarrow e \gamma[85-96,103]$. In left-right symmetric models the LFV decay contribution due to $W_{R}-N_{i}(i=1,2)$ mediation has been computed as early as 1981 assuming a GIM like mechanism in the RH sector [104]. Some of the current experimental bounds on the branching ratios are $\operatorname{Br}\left(\mu^{+} \rightarrow e^{+} \gamma\right)<5.7 \times 10^{-13}, \operatorname{Br}\left(\mu^{+} \rightarrow e^{+} \gamma\right)<$ $5.7 \times 10^{-13}, \operatorname{Br}\left(\mu^{+} \rightarrow e^{+} \gamma\right)<5.7 \times 10^{-13}$, and $\operatorname{Br}\left(\mu^{+} \rightarrow\right.$ $\left.e^{+} \gamma\right)<5.7 \times 10^{-13}$. An up-to-date list of experimental results on various LFV processes and their future projections have been summarised in [103]. In these models contribution to the branching ratios due to the heavier $\mathrm{RH}$ neutrinos is subdominant compared to the lighter singlet fermions

$$
\operatorname{Br}\left(\ell_{\alpha} \longrightarrow \ell_{\beta}+\gamma\right)=\frac{\alpha_{w}^{3} S_{w}^{2} m_{\ell_{\alpha}}^{5}}{256 \pi^{2} M_{W}^{4} \Gamma_{\alpha}}\left|\mathscr{G}_{\alpha \beta}^{N}+\mathscr{G}_{\alpha \beta}^{S}\right|^{2}
$$

where

$$
\begin{aligned}
& \mathscr{G}_{\alpha \beta}^{N}=\sum_{k}\left(\mathscr{V}^{\nu N}\right)_{\alpha k}\left(\mathscr{V}^{\nu N}\right)_{\beta k}^{*} \mathscr{I}\left(\frac{m_{N_{k}}^{2}}{M_{W_{L}}^{2}}\right), \\
& \mathscr{G}_{\alpha \beta}^{S}=\sum_{j}\left(\mathscr{V}^{\nu S}\right)_{\alpha j}\left(\mathscr{V}^{\nu S}\right)_{\beta j}^{*} \mathscr{I}\left(\frac{m_{S_{j}}^{2}}{M_{W_{L}}^{2}}\right), \\
& \mathscr{I}(x)=-\frac{2 x^{3}+5 x^{2}-x}{4(1-x)^{3}}-\frac{3 x^{3} \ln x}{2(1-x)^{4}} .
\end{aligned}
$$

Because of the condition $M_{N} \gg M$, the $\mathrm{RH}$ neutrino exchange contribution is however damped out compared to the sterile fermion singlet contributions. Using allowed solutions, for $M_{1}, M_{2}$, and $M_{3}$, our estimations in the partial degenerate case are given in Table 2 .

As a demonstration of solutions to the conceptual and practical issue of predicting experimentally accessible LFV decay branching ratios consistent with tiny neutrino masses in the dominant seesaw mechanisms (in the event of type I
TABLE 2: Branching ratios for LFV decays $\mu \rightarrow e \gamma, \tau \rightarrow e \gamma$, and $\tau \rightarrow \mu \gamma$ as function of $M_{i}(i=1,2,3)$.

\begin{tabular}{lcccc}
\hline$M_{1,2}(\mathrm{GeV})$ & $M_{3}(\mathrm{GeV})$ & $\mathrm{Br}(\mu \rightarrow e \gamma)$ & $\mathrm{Br}(\tau \rightarrow e \gamma)$ & $\mathrm{Br}(\tau \rightarrow \mu \gamma)$ \\
\hline 1213.11 & 1213.11 & $6.43 \times 10^{-17}$ & $8.0 \times 10^{-16}$ & $2.41 \times 10^{-12}$ \\
500 & 1280 & $1.2 \times 10^{-16}$ & $4.7 \times 10^{-15}$ & $1.45 \times 10^{-12}$ \\
100 & 1319.67 & $1.61 \times 10^{-16}$ & $6.04 \times 10^{-15}$ & $1.80 \times 10^{-12}$ \\
50 & 1545.31 & $9.0 \times 10^{-15}$ & $3.40 \times 10^{-14}$ & $8.0 \times 10^{-12}$ \\
\hline
\end{tabular}

seesaw cancellation) we present results in two specific nonSUSY examples in SO(10): (i) type II dominance [112] and (ii) extended inverse seesaw dominance [110, 175-177]. We have used the Dirac neutrino mass matrices and the fixed value of the matrix $\mu_{S}$ from the respective references. Our predictions for branching ratios as a function of the lightest neutrino mass are shown in in Figure 1 for the type II dominance case. In this figure we have also shown variation of the LH triplet mass as expected from the type II seesaw formula. But in spite of the large value of the triplet mass that normally predicts negligible LFV branching ratios, our model gives experimentally accessible values.

The corresponding results for the extended inverse seesaw case is shown in Figure 2 for the degenerate values of the N-S mixing mass term $M=M_{1}=M_{2}=M_{3}=1.2 \mathrm{TeV}$.

We note that the predictions of LFV decay braching with classic inverse seesaw [24] embedding in non-SUSY SO(10) as investigated in [166] with TeV scale $Z^{\prime}$ boson would be also similar to Figure 2.

5.3. CP Violation due to Nonunitarity. The standard contribution to the $\mathrm{CP}$ violation is determined by the rephasing invariant $J_{\mathrm{CP}}$ associated with the Dirac phase $\delta_{\mathrm{CP}}$ and matrix elements of the PMNS matrix

$$
\begin{aligned}
& J_{\mathrm{CP}} \equiv \operatorname{Im}\left(U_{\alpha i} U_{\beta j} U_{\alpha j}^{*} U_{\beta j}^{*}\right) \\
& =\cos \theta_{12} \cos ^{2} \theta_{13} \cos \theta_{23} \sin \theta_{12} \sin \theta_{13} \sin \theta_{23} \sin \delta_{\mathrm{CP}} .
\end{aligned}
$$

Because of the presence of nonunitarity effects, the leptonic $\mathrm{CP}$ violation can be written as

$$
\mathscr{J}_{\alpha \beta}^{i j}=\operatorname{Im}\left(\mathscr{V}_{\alpha i} \mathscr{V}_{\beta j} \mathscr{V}_{\alpha j}^{*} \mathscr{V}_{\beta j}^{*}\right) \simeq J_{\mathrm{CP}}+\Delta J_{\alpha \beta}^{i j},
$$




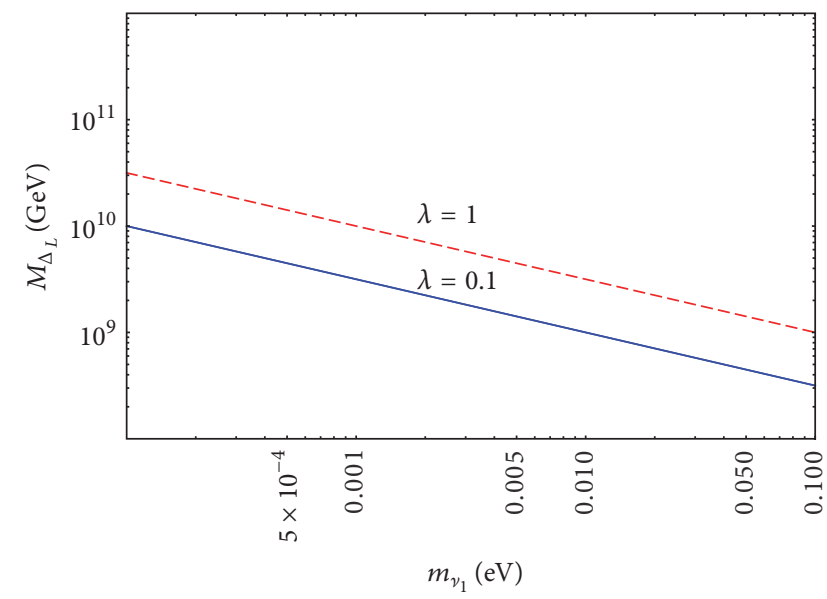

(a)

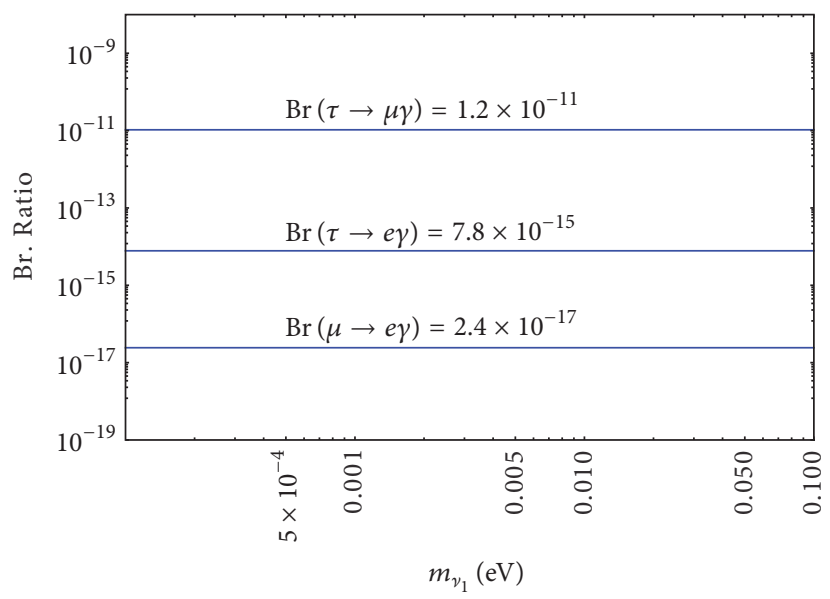

(b)

FIGURE 1: Variation of scalar triplet mass $M_{\Delta_{L}}$ (a) and LFV branching ratio (b) as a function of the lightest neutrino mass for different values of $\lambda$ in a type II seesaw dominant model where we have followed normal ordering. The three almost horizontal lines represent the LFV branching ratios for $M=1.3 \mathrm{TeV}$.

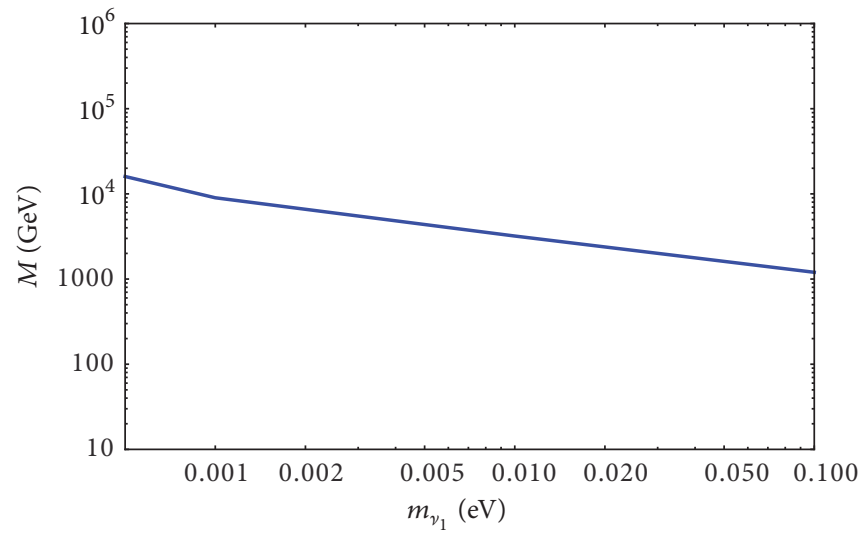

(a)

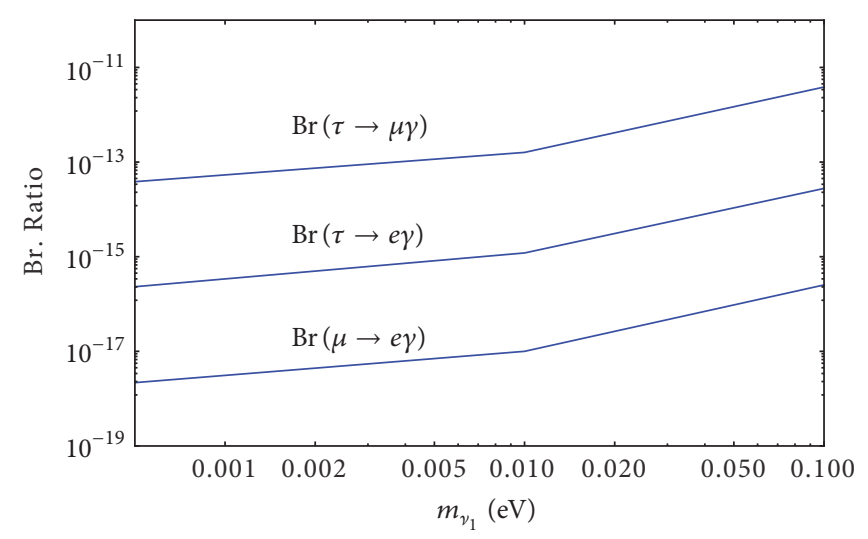

(b)

FIGURE 2: Same as Figure 1 but for extended inverse seesaw dominance. (a) represents variation of $N-S$ mixing mass term $M$ as a function of lightest neutrino mass where we have followed normal ordering. The three lines in (b) represent the LFV branching ratios.

where $[110,112,120-122,164-166,176,218-231]$

$$
\begin{aligned}
& \Delta J_{\alpha \beta}^{i j}=-\sum_{\rho=e, \mu, \tau} \operatorname{Im}\left[\eta_{\alpha \rho} U_{\rho i} U_{\beta j} U_{\alpha j}^{*} U_{\beta i}^{*}\right. \\
& \quad+\eta_{\beta \rho} U_{\alpha i} U_{\rho j} U_{\alpha j}^{*} U_{\beta i}^{*}+\eta_{\alpha \rho}^{*} U_{\alpha i} U_{\beta j} U_{\rho j}^{*} U_{\beta j}^{*} \\
& \left.\quad+\eta_{\beta \rho}^{*} U_{\alpha i} U_{\beta j} U_{\alpha j}^{*} U_{\rho j}^{*}\right] .
\end{aligned}
$$

Model predictions for deviations from rephasing invariant matrix defined in (29) are presented in Table 3 for different allowed values of $M_{i} \equiv m_{R_{i}}$. In order to visualise how far the predicted LFV decay branching ratios are in agreement with neutrino mass values derived from the new seesaw mechanisms which survive after type I cancellation, we have examined two cases as examples: (i) Type II seesaw dominance [112] and (ii) extended seesaw dominance [175-177]. We have used the values of the Dirac neutrino mass matrix and the value of $\mu_{S}$ from these references.

\section{Neutrinoless Double Beta Decay}

6.1. Double Beta Decay Predictions in the $W_{R}-W_{R}$ Channel. The ongoing experiments on double beta decay without any conclusive results have led to a surge of investigations through different theoretical predictions including LR gauge theories [214, 215, 234-236]. While the standard light neutrino exchange amplitude is $\propto G_{F}^{2} \sum_{i} U_{e i}^{2} m_{i}$ in the $W_{L}-W_{L}$ channel, the RH neutrino exchange amplitude in the $W_{R}-W_{R}$ channel is [237-240] $\propto G_{F}^{2}\left[M_{W} / M_{W_{R}}\right]^{4}\left(1 / M_{N}\right)$. Thus the major suppression factor $\left[M_{W} / M_{W_{R}}\right]^{4}<10^{-4}$ apart from the inverse proportionality factor $M_{N}^{-1}$. The $\mathrm{RH}$ triplet exchange contribution is also suppressed [241, 242] and the available 
TABLE 3: Allowed deviations of the rephasing invariant matrix $J_{\mathrm{CP}}$ as a result of weak leptonic CP-violation and nonunitarity effects defined in (29).

\begin{tabular}{lcccccc}
\hline$M_{1,2}(\mathrm{GeV})$ & $M_{3}(\mathrm{GeV})$ & $\Delta \mathscr{F}_{e \mu}^{12}$ & $\Delta \mathscr{F}_{e \mu}^{23}$ & $\Delta \mathscr{J}_{\mu \tau}^{23}$ & $\Delta \mathscr{J}_{\mu \tau}^{31}$ & $\Delta \mathscr{F}_{\tau e}^{12}$ \\
\hline 1213.11 & 1213.11 & $-2.1 \times 10^{-6}$ & $-2.4 \times 10^{-6}$ & $1.4 \times 10^{-4}$ & $1.2 \times 10^{-4}$ & $1.1 \times 10^{-4}$ \\
500 & 1280 & $-2.1 \times 10^{-6}$ & $-2.4 \times 10^{-6}$ & $1.4 \times 10^{-4}$ & $1.2 \times 10^{-4}$ & $1.1 \times 10^{-4}$ \\
100 & 1319.67 & $-2.0 \times 10^{-6}$ & $-3.0 \times 10^{-6}$ & $1.3 \times 10^{-4}$ & $1.8 \times 10^{-4}$ & $3.4 \times 10^{-5}$ \\
50 & 1545.31 & $-1.7 \times 10^{-6}$ & $-1.98 \times 10^{-6}$ & $1.1 \times 10^{-4}$ & $1.8 \times 10^{-4}$ & $8.4 \times 10^{-5}$ \\
\hline
\end{tabular}

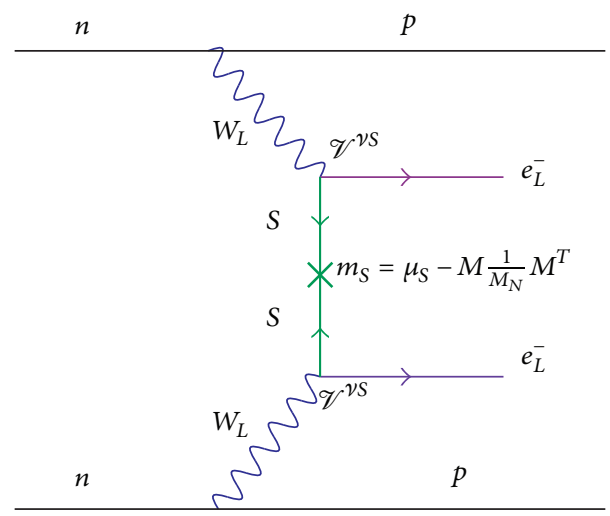

Figure 3: Feynman diagram for neutrinoless double beta decay due to exchange of light sterile neutrino $S$.

experimental limit on the double beta decay rates has led to the lower bound on the doubly charged Higgs mass [234, 235]

$$
M_{\Delta_{R}^{++}} \geq 500\left(\frac{3.5 \mathrm{TeV}}{M_{W_{R}}}\right)^{2} \times\left(\frac{M_{N}}{3 \mathrm{TeV}}\right)^{1 / 2}
$$

Possible dominant contribution [175] in the $W_{L}-W_{R}$ channel has been also suggested due to what are known as $\eta$ and $\lambda$ diagrams [214, 215]. It has been concluded [9] that the lack of observation of double beta decay cannot rule out $\mathrm{TeV}$ scale LR models.

6.2. Double Beta Decay Predictions in the $W_{L}-W_{L}$ Channel. In [176] it was noted in the context of extended gauged inverse seesaw mechanism in non-SUSY $S O(10)$ that a new and dominant contribution to $0 \nu \beta \beta$ decay exists through lefthanded weak charged currents in the $W_{L}-W_{L}$ channel via exchanges of fermion singlets $S_{i}$. That the light sterile neutrinos may have a dominant effect was also noted in the context of extensions of SM or LR models [48] where type I contributions to neutrino mass were also included. As such this model [48] did not use cancellation mechanism for the type I seesaw contributions. The sterile fermions mix quite prominently through Dirac neutrino mass matrix which is well known to be of order of up quark mass matrix. As already noted the sterile neutrinos acquire Majorana masses $m_{S} \simeq$ $\mu_{S}-M\left(1 / M_{N}\right) M^{T} \simeq-M\left(1 / M_{N}\right) M^{T}$. The Feynman diagram for double beta decay due to light sterile neutrino exchange is shown in Figure 3.
The (ei) element of the $\nu$-S mixing matrix is

$$
\mathscr{V}_{e i}^{\nu S}=\left(\frac{M_{D}}{M}\right)_{e i},
$$

where the Dirac neutrino mass matrix $M_{D}$ has been given in different $S O(10)$ models from fits to the charged fermion masses at the GUT scale and then running it down to the TeV scale in non-SUSY SO(10) models [110, 112, 166, 176, 177]. For example the Dirac neutrino mass matrix $M_{D}$ in the model [112] is

$$
\begin{aligned}
& M_{D}(\mathrm{GeV}) \\
& \quad=\left(\begin{array}{ccc}
0.014 & 0.04-0.01 i & 0.109-0.3 i \\
0.04+0.01 i & 0.35 & 2.6+0.0007 i \\
0.1+0.3 i & 2.6-0.0007 i & 79.20
\end{array}\right) .
\end{aligned}
$$

The elements of the matrix $M$ treated as diagonal are determined from constraints on LFV decays and the diagonal elements are estimated using the nonunitarity equation as discussed in the previous section. We derive the relevant elements of the mixing matrix $\mathscr{V}^{\nu S}$ using the structures of the Dirac neutrino mass matrix $M_{D}$ given in (32) and values of the diagonal elements of $M=\left(M_{1}, M_{2}, M_{3}\right)$ satisfying the nonunitarity constraint in (23). The eigenvalues of the $S$-fermion mass matrix are estimated for different cases using the structures of the $\mathrm{RH}$ Majorana neutrino mass matrices [112, 175-177] and allowed values of $M_{i}$ satisfying the nonunitarity constraints through the formula $M_{S}=$ $-M\left(1 / M_{N}\right) M^{T}$.

Different particle exchange contributions for $0 \nu 2 \beta$ decay are changed by the chirality of the hadronic currents involved in the nuclear mass matrix element. In this model only the LH currents are significant or dominant while the RH current effects are negligible. The inverse half-life has been estimated with proper normalization factors by taking into account the effects of nuclear matrix elements [214, 215, 237, 243-253]

$$
\begin{aligned}
{\left[T_{1 / 2}^{0 \nu}\right]^{-1} } & \simeq G_{01}^{0 \nu}\left|\frac{\mathscr{M}_{\nu}^{0 \nu}}{m_{e}}\right|^{2}\left|\left(\mathbf{m}_{\nu}^{e e}+\mathbf{m}_{S}^{e e}+\mathbf{m}_{N}^{e e}\right)\right|^{2} \\
& \simeq K_{0 \nu}\left|\left(\mathbf{m}_{\nu}^{e e}+\mathbf{m}_{S}^{e e}\right)\right|^{2} .
\end{aligned}
$$

The larger value of RH neutrino masses makes negligible contribution to the $0 \nu \beta \beta$ decay amplitude. In the above equation $G_{01}^{0 \nu}=0.686 \times 10^{-14} \mathrm{yrs}^{-1}, \mathscr{M}_{\nu}^{0 \nu}=2.58-6.64$, 


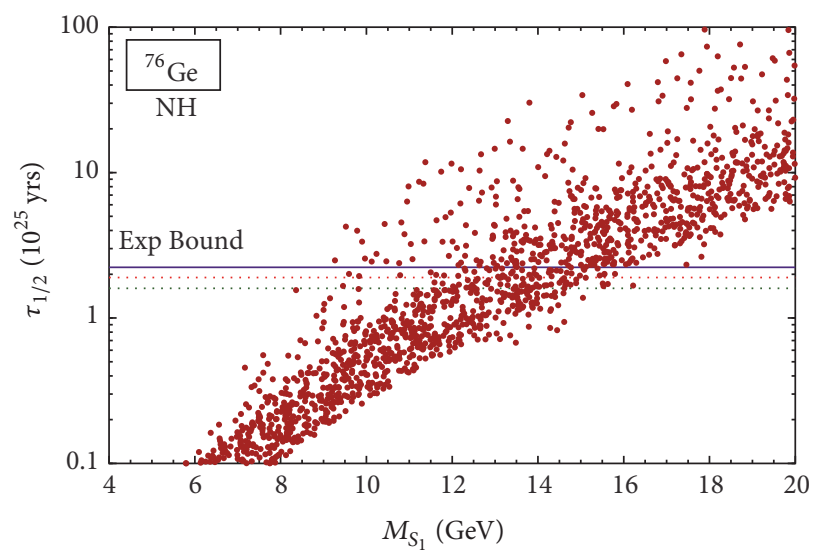

(a)

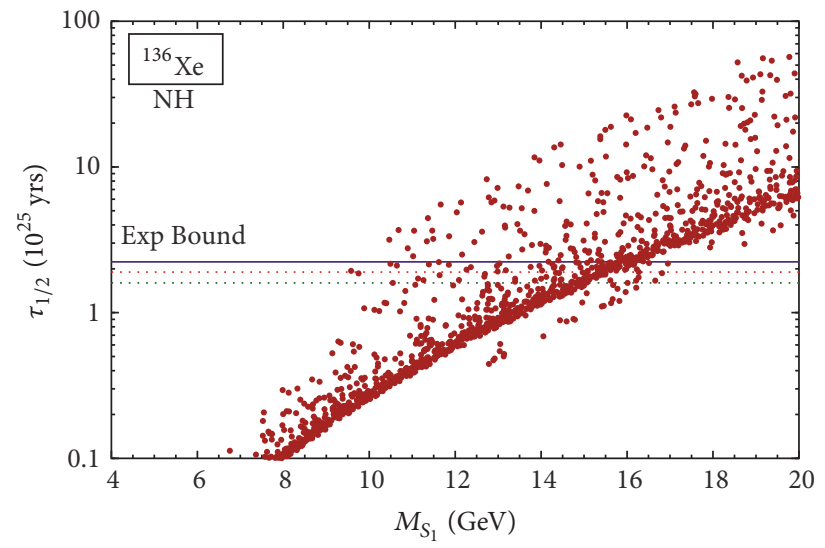

(b)

FIGURE 4: Scattered plots for half-life prediction of double beta decay as a function of neutral singlet fermion mass in the case of normal hierarchy $(\mathrm{NH})$ of light neutrino masses from ${ }^{76} \mathrm{Ge}$ nucleus (a) and from ${ }^{136} \mathrm{Xe}$ nucleus (b).

$K_{0 v}=1.57 \times 10^{-25} \mathrm{yrs}^{-1} \mathrm{eV}^{-2}$, and the two effective mass parameters are

$$
\begin{aligned}
& \mathbf{m}_{v}^{e e}=\sum_{i}\left(\mathscr{V}_{e i}^{v v}\right)^{2} m_{v}, \\
& \mathbf{m}_{S}^{e e}=\sum_{i}\left(\mathscr{V}_{e i}^{\nu S}\right)^{2} \frac{|p|^{2}}{m_{S_{i}}}, \\
& \mathbf{m}_{N}^{e e}=\sum_{i}\left(\mathscr{V}_{e i}^{v N}\right)^{2} \frac{|p|^{2}}{m_{N_{i}}} .
\end{aligned}
$$

Here $m_{S_{i}}$ is the eigenvalue of the $S$-fermion mass

$$
M_{S}=-M \frac{1}{M_{N}} M^{T}
$$

6.3. Singlet Fermion Exchange Dominated Half-Life. The nonstandard contributions to half-life of double beta decay as a function of sterile neutrino mass has been discussed in $[110,112,166,176,177]$. The models predict $\nu$-S mixing and sterile neutrino masses which have been used to predict the half-life in the case of different hierarchies of light neutrino masses: NH, IH, and QD. Using the estimations of [177] which are also applicable to models of $[110,112,175,176]$, scattered plots for the predicted half-life are shown in Figures 4,5 , and 6 for the three types of neutrino mass hierarchies.

In Figure 7 we show predictions in the QD case excluding and including the $\mathrm{CP}$ phases of Majorana type sterile neutrinos. The cancellations between light neutrino exchange amplitude and the sterile neutrino exchange amplitude are shown by the two peaks. When CP phases associated with the Majorana type sterile neutrino mass eigenvalue(s) are included the peaks are smoothened as shown by dotted lines [177].

In Figure 8 estimations on the lightest sterile neutrino masses are predicted which saturate the current experimental limit on the observed double beta decay half-life using $\mathrm{Ge}$ 76 and Xe-136 nuclei for three different light neutrino mass hierarchies in each case. The uncertainties in the predicted masses correspond to the existing uncertainty in the neutrino virtuality momentum $|p|=120-200 \mathrm{MeV}$. The green horizontal line represents the average value

$$
\widehat{M}_{S_{1}}=18 \pm 4 \mathrm{GeV}
$$

of the lightest sterile neutrino mass determined from double beta decay experimental bound [177]. Lower values of this mass have been obtained using light neutrino assisted type II seesaw dominance [112].

These predictions suggest that sterile neutrino exchange contribution dominates the double beta decay rate even when the light neutrino masses have $\mathrm{NH}$ or $\mathrm{IH}$ type of mass hierarchies. To predict double beta decay saturating the current experimental bounds, it is not necessary that light neutrinos should be quasi-degenerate in mass. On the other hand if double beta decay is not found with half-life close to the current limits, then the solutions with light sterile neutrino masses in the range $\sim(2-15) \mathrm{GeV}$ are ruled out, but the model with larger mass eigenvalues easily survives.

\section{Leptogenesis in Extended MSSM and SUSY $S O(10)$}

In the conventional type I seesaw based leptogenesis models where heavy $\mathrm{RH}$ neutrino decays give rise to the desired lepton asymmetry [254], the Davidson-Ibarra bound [255, 256] imposes a lower limit on the scale of leptogenesis, $M_{N_{1}}>4.5 \times 10^{9} \mathrm{GeV}[255,256]$. This also suggests the lower bound for the reheating temperature after inflation, $T_{\mathrm{RH}} \geq 10^{9} \mathrm{GeV}$, that would lead to overproduction of gravitino severely affecting the relic abundance of light nuclei since the acceptable limit has been set as $T_{\mathrm{RH}} \leq$ $10^{7} \mathrm{GeV}$ [257]. Several attempts have been made to evade the gravitino constraint on leptogenesis where sterile neutrino assisted results are interesting. Obviously gravitino constraint is satisfied in models with $\mathrm{TeV}$ scale resonant leptogenesis [258]. Also in the singlet fermion extended SUSY SO(10) 


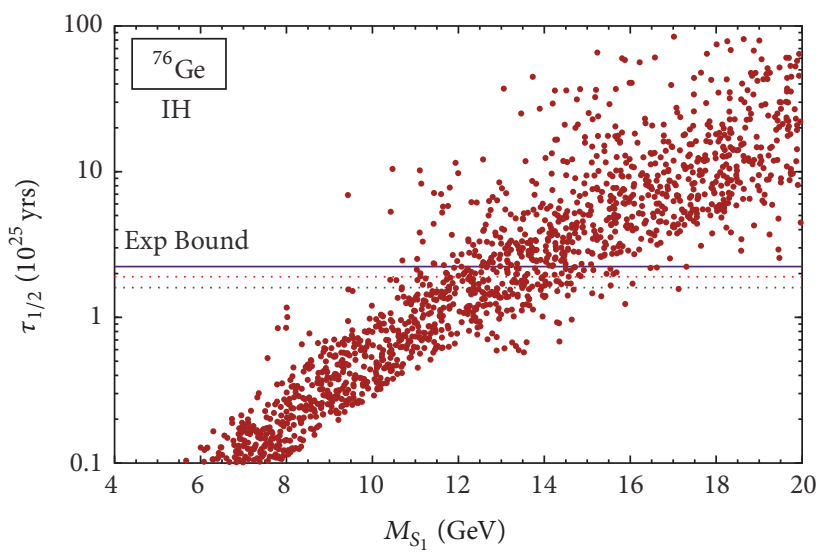

(a)

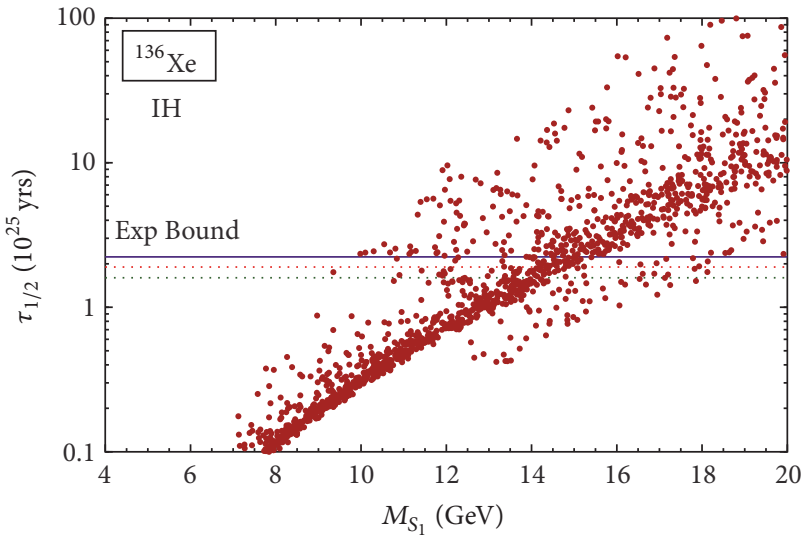

(b)

FIGURE 5: Same as Figure 4 but for inverted hierarchy (IH) of light neutrino masses.

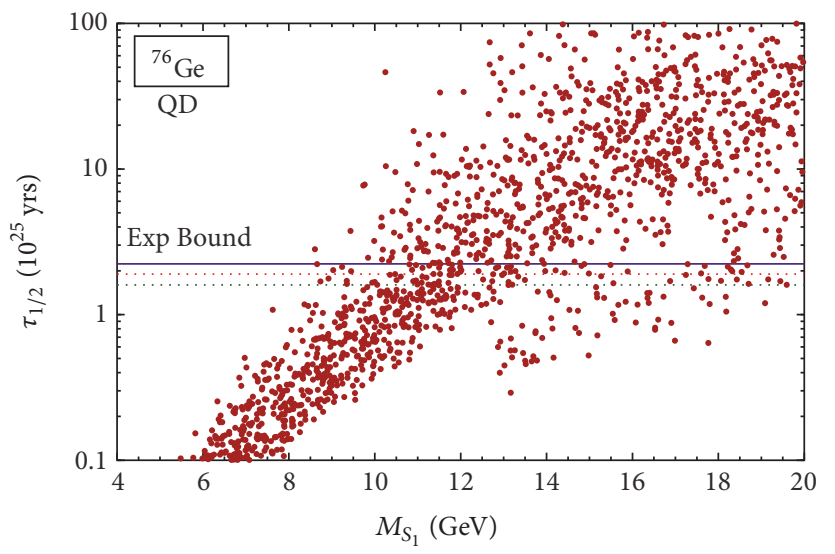

(a)

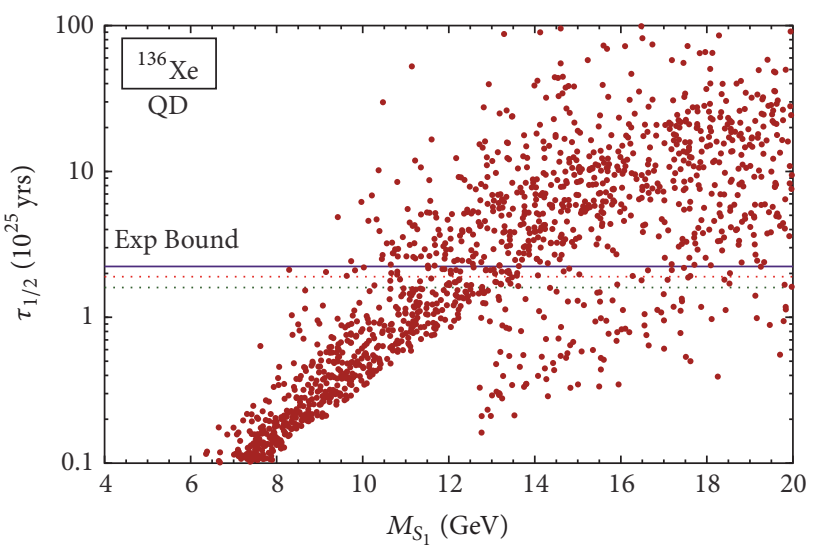

(b)

FIGURE 6: Same as Figure 4 but for quasi-degenerate (QD) type light neutrino masses.

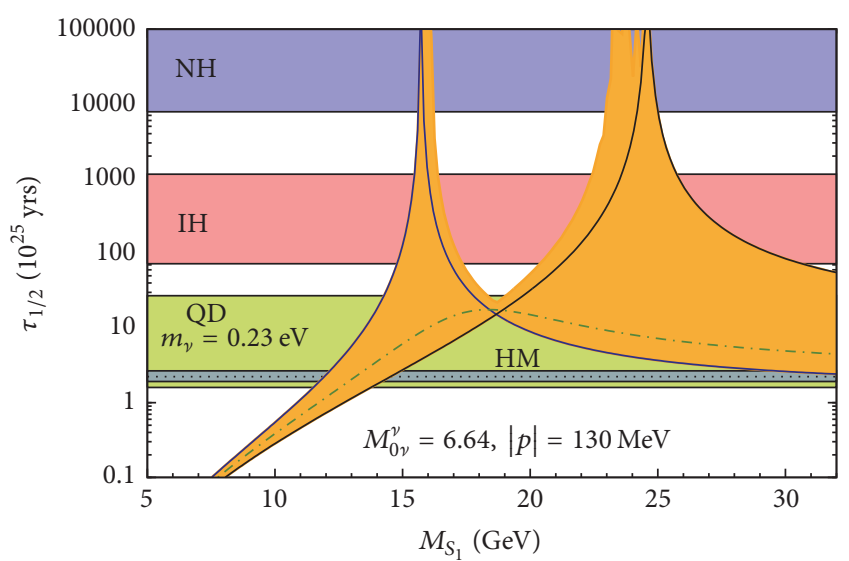

FIGURE 7: Prediction of half-life for double beta decay as a function of sterile neutrino mass in the case of QD type mass hierarchy with the common mass parameter $m_{0}=0.23 \mathrm{eV}$. The peaks correspond to cancellation between light neutrino and sterile neutrino exchange amplitudes when Majorana CP phases of sterile neutrino are ignored. The dotted line shows the absence of peaks when CP phases are included [177]. where $\mathrm{RH}$ neutrinos are heavy pseudo Dirac neutrinos and neutrino mass formula is through inverse seesaw [24], there is no problem due to gravitino constraint $[164,165,199]$. We discuss the cases where all the three types of neutrinos are Majorana fermions.

7.1. Leptogenesis with Extended Seesaw Dominance. With extended seesaw realisation of leptogenesis two types of SUSY models have been investigated under gravitino constraint: (i) MSSM extension with fermion singlets [45, 46, 169-171] and (ii) Singlet extension of $S O(10)$ with intermediate scale $G_{2213}$ gauge symmetry $[172,173]$. We discuss their salient features.

7.1.1. MSSM Extension with Fermion Singlets. The Dirac neutrino mass matrix is identified with the charged lepton mass matrix in this model where MSSM is extended with the addition of heavy $\mathrm{RH}$ neutrinos $N_{i}$ as well as additional singlets $S_{i}[45,46]$, one for each generation. As already explained in the limit $M_{N}>M \gg M_{D}, \mu_{S}$ extended seesaw formula, which is the same as the inverse seesaw formula 


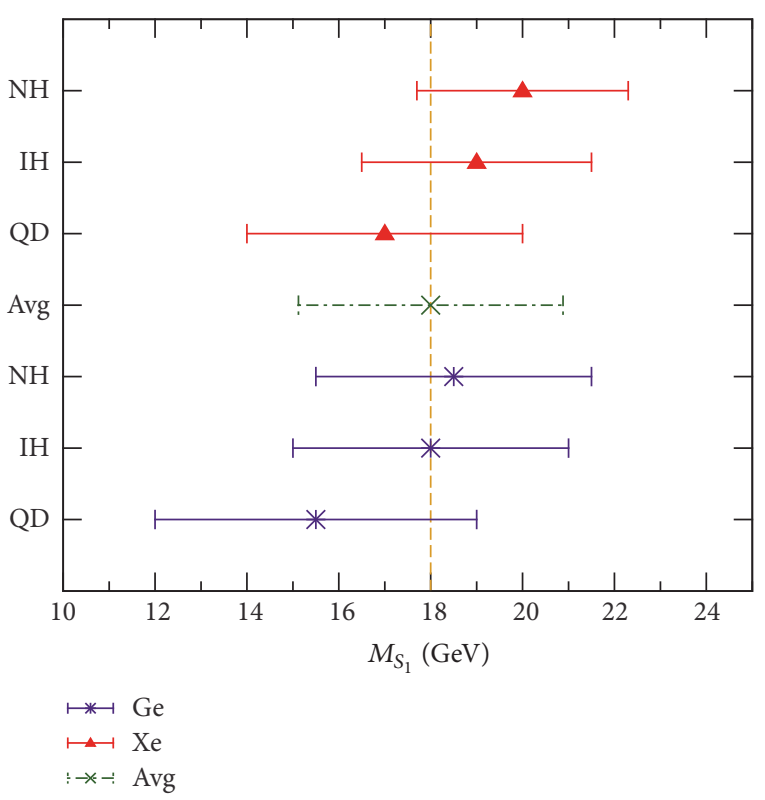

FIgURE 8: Prediction of light sterile neutrino mass from the saturation of experimental decay rates of ongoing searches for different active neutrino mass hierarchies. The horizontal green line indicates the average value of all results.

for active neutrino mass. In this case resonant leptogenesis is implemented via quasi-degenerate $\mathrm{RH}$ neutrino decays at the TeV scale. It is well known that such resonant leptogenesis scenario with $M_{N_{1}} \sim M_{N_{2}} \sim 1 \mathrm{TeV}$ implemented through canonical seesaw needs a very small mass splitting between the RH neutrinos $\left(M_{N_{2}}-M_{N_{1}}\right) /\left(M_{N_{2}}+M_{N_{1}}\right) \sim 10^{-6}$. With the tension arising out of fitting the neutrino oscillation data being transferred from type I seesaw to extended seesaw in the presence of additional sterile fermions, it is not unimaginable that this fine-tuning associated with very tiny $\mathrm{RH}$ neutrino mass splitting could be adequately alleviated. The fermion singlets $S_{i}$ give rise to a new self-energy contribution and using this successful resonant leptogenesis has been found to be possible for a much larger mass ratio $M_{N_{2}} / M_{N_{1}} \sim$ 10. Possibilities of $\sim 100 \mathrm{MeV}$ to $\sim 10 \mathrm{GeV}$ mass range for light sterile neutrinos have been pointed out. In a separate analysis the possibility of singlet Majorana fermion or singlet scalar as candidates of dark matter has been pointed out [169]. Realisation of doubly coexisting dark matter candidates in the context of extended seesaw framework has been pointed out [170].

7.1.2. Leptogenesis in SUSY SO(10). In non-SUSY minimal LR models where $M_{D}$ is similar to charged lepton mass matrix successful leptogenesis emerges with intermediate scale hierarchical RH neutrino masses [259]. In SUSY SO(10) the underlying quark-lepton unification forces the Dirac neutrino mass to be similar to the up quark mass matrix. This pushes the type I seesaw scale closer to the GUT scale $M_{R} \geq$ $10^{14} \mathrm{GeV}$ and rules out the possibility of low scale $W_{R}$ bosons accessible to accelerator searches in foreseeable future unless the canonical seesaw ansatz is given up, for example, in favour of inverse seesaw with $\mathrm{TeV}$ scale pseudo Dirac neutrinos and $W_{R}$ bosons $[164,165,199]$. With heavy right-handed Majorana neutrinos and GUT scale LR breaking scale, successful leptogenesis has been implemented in realistic SUSY SO(10) [260]. With the help of an effective dim.5 operator ansatz which originates from renormalisable interactions at GUTPlanck scale in SUSY SO(10) (without using 126 $6_{H}$ ) both thermal and nonthermal leptogenesis [261-264] have been discussed with heavy hierarchical $\mathrm{RH}$ neutrino of masses $[265,266]$. Possible solutions to the allowed parameter space to evade gravitino constraint have been also discussed in this work.

Apart from the models with resonant leptogenesis, possibility of leptogenesis under gravitino constraint in SUSY $\mathrm{SO}(10)$ has been realised with hierarchical $\mathrm{RH}$ neutrinos assisted by sterile neutrinos. As already noted above, in these cases the extended seesaw formula controls the neutrino mass as a result of cancellation of type I seesaw contribution. Gauge coupling unification in these $S O(10)$ models requires the $G_{2213}$ symmetry to occur at the intermediate scale in the renormalisable model [173]. A common feature of both these models $[172,173]$ is the generation of lepton asymmetry through the decay of hierarchical sterile neutrinos through their respective mixings with heavier $\mathrm{RH}$ neutrinos which are also hierarchical.

An important and specific advantage of heavy gauge singlet neutrino decay to achieve leptonic CP asymmetry is the following: The singlet neutrino of mass $\sim 10^{5} \mathrm{GeV}$ which decays to $l \phi$ though its mixing with $\mathrm{RH}$ neutrino of mass $\sim 10^{10} \mathrm{GeV}$ has a small mixing angle $\sim 10^{-5}$. This small mixing ensures out-of-equilibrium condition by making the decay rate smaller than the Hubble expansion rate in arriving at $\mathrm{CP}$ asymmetry at lower temperatures $\sim 300 \mathrm{GeV}$.

\section{Singlet Fermion Assisted Leptogenesis in Non-SUSY $S O(10)$}

An extensive review of thermal leptogenesis with reference to LFV is available in [267]. With the neutrino mass following a modified type I seesaw at a scale $\geq 10^{8} \mathrm{GeV}$, thermal leptogenesis has been investigated in [267-273]. It is well known that $\mathrm{TeV}$ scale $\mathrm{RH}$ neutrinos can participate in resonant leptogenesis contributing to enhanced generation of leptonic CP asymmetry that is central to generation of baryon asymmetry of the universe via sphaleron interactions. Here we briefly discuss a recent work where quasi-degenerate sterile neutrinos at the TeV scale in non-SUSY SO(10) have been shown to achieve resonant leptogenesis through their decays. The Feynman diagrams at the tree level and with vertex and self-energy corrections are shown in Figure 9.

The fermion-Higgs coupling in all the diagrams is $V h$ instead of the standard Higgs-Yukawa coupling $h=M_{D} / V_{\mathrm{wk}}$, where $\mathscr{V} \simeq M / M_{N}, M_{D}$ is given in (32), and $V_{\mathrm{wk}} \simeq$ $174 \mathrm{GeV}$. Denoting the mass eigenvalue of a sterile neutrino by $\widehat{m}_{S_{k}}(k=1,2,3)$, for computation of baryon asymmetry $Y_{B}$ of the Universe with a washout factor $K_{k}$, we utilise the ansatz [124] 

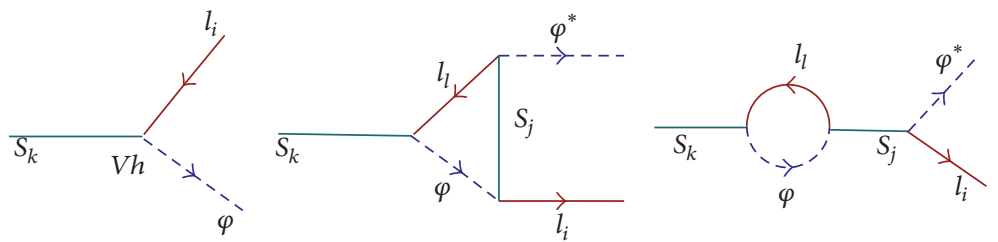

Figure 9: Tree and one-loop diagrams for the $S_{k}$ decay contributing to the CP asymmetry. All fermion-Higgs couplings in the diagrams are of the form $V h$ where $h=N-l-\Phi$ Yukawa coupling and $V \simeq M / M_{N}$.

$$
\begin{aligned}
Y_{B} & \simeq \frac{\varepsilon_{S_{k}}}{200 K_{k}}, \\
K_{k} & =\frac{\Gamma_{S_{k}}}{H\left(\widehat{m}_{S_{k}}\right)},
\end{aligned}
$$

$H\left(\widehat{m}_{S_{k}}\right)$ being the Hubble parameter at temperature $\widehat{m}_{S_{k}}$. Defining

$$
\delta_{i}=\frac{\left|\widehat{m}_{S_{i}}-\widehat{m}_{S_{j}}\right|}{\Gamma_{S_{i}}} \quad(i \neq j),
$$

the depleted washout factor is [274]

$$
K_{i}^{\mathrm{eff}} \simeq \delta_{i}^{2} K_{i}
$$

Here we discuss two cases for the sterile neutrino contribution towards leptogenesis and baryon asymmetry: (1) $\widehat{m}_{S_{1}}$ is light, $\widehat{m}_{S_{2}}$ and $\widehat{m}_{S_{3}}$ are quasi-degenerate; (2) $\widehat{m}_{S_{2}}$ is light, $\widehat{m}_{S_{1}}$ and $\widehat{m}_{S_{3}}$ are quasi-degenerate.

Case $1\left(\widehat{m}_{S_{1}}\right.$ light, $\widehat{m}_{S_{2}}$ and $\widehat{m}_{S_{3}}$ heavy and quasi-degenerate). Using an allowed interesting region of the parameter space $M \simeq \operatorname{diag}(146,3500,3500) \mathrm{GeV}, V_{R}=10^{4} \mathrm{GeV}$, and $M_{N}=$ $f V_{R}$ we get

$$
\widehat{m}_{S_{i}}=\operatorname{diag}(1.0,595.864 . ., 595.864 . .) \mathrm{GeV} \text {, }
$$

leading to $K_{2}=2.7 \times 10^{7}$. Using $\left(\widehat{m}_{S_{2}}-\widehat{m}_{S_{3}}\right) \simeq 2 \times 10^{-7} \mathrm{GeV}$, we obtain

$$
\begin{aligned}
& \varepsilon_{S_{2}}=0.824, \\
& Y_{B}=1.5 \times 10^{-10} .
\end{aligned}
$$

Case $2\left(\widehat{m}_{S_{2}}\right.$ light, $\widehat{m}_{S_{1}}$ and $\widehat{m}_{S_{3}}$ heavy and quasi-degenerate). Choosing another allowed region of the parameter space $M \simeq$ $\operatorname{diag}(3200,146,3200) \mathrm{GeV}$, similarly we get

$$
\widehat{m}_{S_{i}}=\operatorname{diag}(500.567 . ., 1.0,500.567 . .) \mathrm{GeV} \text {, }
$$

leading to $K_{1}=4 \times 10^{6}$. using $\left(\widehat{m}_{S_{1}}-\widehat{m}_{S_{3}}\right) \simeq 7 \times 10^{-5} \mathrm{GeV}$, we obtain

$$
\begin{gathered}
\varepsilon_{S_{1}}=0.682, \\
Y_{B}=4 \times 10^{-10} .
\end{gathered}
$$

In Case 1 with $\widehat{m}_{S_{1}} \sim \mathcal{O}(1) \mathrm{GeV}$, the lightest sterile neutrino acts as the most dominant source of $0 \nu \beta \beta$ decay
TABle 4: Predictions for baryon asymmetry and double beta decay half-life as a function of sterile neutrino masses.

\begin{tabular}{lcccc}
\hline $\begin{array}{l}m_{S_{1}} \\
(\mathrm{GeV})\end{array}$ & $m_{S_{2}}(\mathrm{GeV})$ & $m_{S_{3}}(\mathrm{GeV})$ & $\begin{array}{c}\text { Baryon } \\
\text { asymmetry }\end{array}$ & $\begin{array}{c}T_{1 / 2}^{0 \nu} \\
\left(10^{25} \mathrm{yrs} .\right)\end{array}$ \\
\hline 1 & 500 & 500 & $3.73 \times 10^{-10}$ & 2.72 \\
10 & 500 & 500 & $3.5 \times 10^{-10}$ & 16.01 \\
500 & 1 & 500 & $4.2 \times 10^{-10}$ & 0.0494 \\
500 & 3 & 500 & $4.1 \times 10^{-10}$ & 2.19 \\
\hline
\end{tabular}

whereas the heavy quasi-degenerate pair of sterile neutrinos $S_{2}$ and $S_{3}$ mediate resonant leptogenesis. Similarly in the alternative scenario of Case 2 with $\widehat{m}_{S_{2}} \sim \mathcal{O}(1) \mathrm{GeV}$, the second generation light sterile neutrino acts as the mediator of dominant double beta decay while the heavy quasidegenerate pair of the first and the third-generation sterile neutrinos mediate resonant leptogenesis. Because of the resonant leptogenesis constraint, we note that either Case 1 or Case 2 is permitted, but not both.

Our predictions for the double beta decay half-life and the baryon asymmetry in Cases 1 and 2 are presented in Table 4. It is clear that for smaller mass eigenvalues of sterile neutrinos in Case 1 or Case 2 it is possible to saturate current experimental limit on the double beta decay half-life while explaining the right order of magnitude of the baryon asymmetry. Thus, in addition to the Case 1 found in [112], we have shown another possible alternative scenario as Case 2.

Before concluding this section certain interesting results on thermal leptogenesis derived earlier are noted. Thermal leptogenesis with a hybrid seesaw and $\mathrm{RH}$ neutrino dark matter have been proposed by introducing additional $U(1)$ gauge symmetry [136]. Thermal leptogenesis in extended seesaw models has been investigated earlier [268-272] which are different from our cases reported here and earlier [112, 113]. Possibilities of falsifying high scale leptogenesis on the basis of certain LHC results and also on the basis of LFV and $0 \nu \beta \beta$ decay results have been suggested $[273,275]$. Prospects of dark matter in the minimal inverse seesaw model have been also investigated in ref. [276, 277].

\section{Summary and Discussion}

Reviewing the contributions already made, we have discussed how the added presence of singlet fermions which manifest as singlet neutrinos of Majorana type effectively cancels out the would-be dominant type I seesaw contribution to light 
neutrino masses. The neutrino masses consistent with the oscillation data are now governed by the classic inverse seesaw formula (renamed as extended seesaw formula) or type II seesaw, or even linear or double seesaw under their respective limiting conditions. But the dominant part of the singlet neutrino mass is given by the type I seesaw mechanism where the role of the Dirac neutrino mass matrix $M_{D}$ is replaced by the $N-S$ mixing matrix $M$. The cancellation mechanism of type I seesaw term for the active light neutrino masses is universal in the sense that appropriate extensions of SM, left-right gauge theories, Pati-Salam Model, SO(10), SUSY, or non-SUSY can accommodate it leading to the dominance of seesaw mechanism of another type. In the cases of extended seesaw, linear seesaw, or type II seesaw dominance, the double beta decay rates in the $W_{L}-W_{L}$ channel are dominated by the exchange of light sterile neutrinos with masses in the range of $\mathcal{O}(1-10) \mathrm{GeV}$. With type II dominance, the second- and third-generation sterile neutrinos could be heavy and quasidegenerate and explain baryon asymmetry of the universe through resonant leptogenesis. The models also predict a rich variety of results for LFV decays and leptonic nonunitarity effects. In SUSY GUTs in the absence of added fermion singlets baryogenesis via leptogenesis through decays of heavy RH neutrinos is usually affected by gravitino problem. A possible solution to this is the well known $\mathrm{TeV}$ scale resonant leptogenesis which requires extremely small mass difference between the heavy quasi-degenerate pair leading to their unity mass ratio up to very high degree of accuracy. The presence of sterile neutrinos considerably alleviates this problem by changing this fine-tuned mass ratio to a value as large as $\sim 10$. In SUSY SO(10) leptogenesis under gravitino constraint is achieved even for large hierarchical masses of $\mathrm{RH}$ neutrinos. When the model is extended by fermion singlets, the singlet neutrinos which mix with $\mathrm{RH}$ neutrinos also acquire hierarchical masses below $10^{6} \mathrm{GeV}$. The hierarchical singlet neutrinos decay through their mixings with $\mathrm{RH}$ neutrinos to generate the desired leptonic CP asymmetry. In these models also the light neutrino mass formula is due to the extended seesaw (or inverse seesaw). One simple reason for the success of the sterile neutrino assisted leptogenesis in SUSY SO(10) is the smallness of mixing angle $\xi$ between the lighter sterile neutrino and the heavy $\mathrm{RH}$ neutrino with $\sin \xi \sim M / M_{N} \sim 10^{-5} \rightarrow 10^{-6}$. This reduces the decay rate of the sterile neutrino considerably to satisfy the out-of-equilibrium condition which forms an important ingredient for the generation of CP asymmetry.

In the presence of sterile neutrinos, type II seesaw dominance is achieved with $U(1)_{B=L}$ breaking scale much lower than the mass of the Higgs triplet $\Delta_{L}(3,1,-2,1)$. This mechanism makes it possible to have type II seesaw formula even with $\mathrm{TeV}$ scale of $W_{R}$ or $Z_{R}$ boson whereas in the conventional attempts in SUSY or non-SUSY SO(10), or split-SUSY models, the type II seesaw dominance required these $W_{R}$ and $Z_{R}$ boson masses to be near the GUT-Planck scale. In the standard lore in the proposed models of type II seesaw dominance with very large $W_{R}$ and $\mathrm{RH}$ neutrino masses, the nonstandard contribution to double beta decay in the $W_{L}-W_{L}$ channel, damped by the heavy $\Delta_{L}(3,1,-2,1)$ boson propagator, is negligible. But this concept is overthrown when type II seesaw dominance is assisted by sterile neutrinos. In the new scheme even though the heavy $\Delta_{L}(3,1,-2,1)$ boson exchange contribution is negligible, the double beta decay mediation by the exchange of light sterile neutrinos in the $W_{L}-W_{L}$ channel predicts dominant decay rates saturating the current experimental limits. This new model of type II seesaw dominance predicts resonant leptogenesis in the non-SUSY $S O(10)$ originating from the near $\mathrm{TeV}$ scale masses of quasidegenerate sterile neutrinos of the other two generations.

While GUTs like SUSY SO(10) have a rich structure of dark matter candidates, even in the presence of sterile neutrinos, some aspects of embedding dark matter and their detection possibilities in the SM extensions have been discussed in [169-171]. The SUSY and non-SUSY SO(10) GUTs considered under these seesaw mechanisms satisfy coupling unification and proton life time constraints, the latter being accessible to ongoing search experiments $[109,110,112$, 173, 176, 177].

In this review we have considered the class of models where heavy RH Majorana mass terms are present satisfying the conditions $M_{N}>M \gg M_{D}, \mu_{S}$ under which the generalised form of the neutral fermion matrix gives different seesaw formulas. In these models the type I seesaw, if not cancelled by using the decoupling criteria and two-step block diagonalisation process, would have given dominant contributions. A common feature of all these models is dominant double beta decay in the $W_{L}-W_{L}$ channel mediated by light sterile neutrinos as well as leptogenesis generated by heavier sterile neutrinos of the other two generations.

The singlet neutrinos needed for these models are found to have mass ranges between few $\mathrm{GeV}$ to $\sim 1 \mathrm{TeV}$. They are the mixed states of added fermion singlets $S_{i}$ and heavy $\mathrm{RH}$ neutrinos $N_{i}$ where the latter are in the spinorial representation of $S O(10)$. The mass terms of fermion singlets violate the global lepton number symmetry of the SM. As such these masses are required to be as light as possible according to 't Hooft's naturalness criteria [163]. In other words the global lepton number symmetry protects these masses naturally and prevents them from becoming superheavy. This is a special advantage in favour of $\mathrm{TeV}$ scale seesaw mechanisms as well as the new type II intermediate scale seesaw mechanism [112] due to the cancellation of the type I seesaw. Further we have brought down the $U(1)_{R} \times U(1)$ breaking scale in nonSUSY SO(10) to be accessible to LHC and future accelerator searches by a number of new physical processes including the $Z^{\prime}$ boson. The predicted proton lifetime has been noted to be accessible to ongoing searches [278-280].

There are a number of interesting models assisted by $\mathrm{TeV}$ scale pseudo Dirac neutrinos [109, 164-168, 199] where such heavy RH Majorana neutrino masses are either absent or, if present, they do not satisfy the decoupling criteria. Details of phenomenology and predictions of such models are beyond the scope of the present review. Likewise the interesting possibilities of detection of gauge singlet neutrinos through their displaced vertices [113, 117-119] and the renormalisation group impacts [281] in the presence of inverse seesaw have been excluded from present discussions. 


\section{Competing Interests}

While submitting this manuscript for publication, the authors declare that they have no conflict of interests with any individual or organisation, private or governmental, whatsoever.

\section{Acknowledgments}

The authors are thankful to Professor R. N. Mohapatra and Dr. Mainak Chakraborty for discussions. M. K. Parida acknowledges research Grant no. SB/S2/HEP-011/2013 from the Department of Science and Technology, Govt. of India. Bidyut Prava Nayak acknowledges a junior research fellowship from the Siksha 'O' Anusandhan University, Bhubaneswar.

\section{References}

[1] R. N. Mohapatra and A. Yu. Smirnov, "Neutrino mass and new physics," Annual Review of Nuclear and Particle Science, vol. 56, pp. 569-628, 2006.

[2] R. N. Mohapatra, "Neutrino mass and the origin of matter," Physics Today, vol. 63, no. 4, pp. 68-69, 2010.

[3] G. Senjanovic, "Neutrino mass: from LHC to grand unification," La Rivista del Nuovo Cimento, vol. 34, pp. 1-68, 2011.

[4] W. Grimus, "Theory of neutrino masses and mixing," Physics of Particles and Nuclei, vol. 42, no. 4, pp. 566-576, 2011.

[5] G. Altarelli, "Neutrinos today: an introduction," in Proceedings of the 49th Recontres de Moriond on Electroweak Interactons and Unified Theories, pp. 15-22, Thuile, Italy, March 2014.

[6] A. Y. Smirnov, “Theories of neutrino masses and mixings," Il Nuovo Cimento, vol. 37, no. 3, pp. 29-37, 2014.

[7] G. Senjanovic and V. Tello, "Origin of neutrino mass," PoS PLANCK2015, vol. 141, 2016.

[8] R. N. Mohapatra, "From old symmetries to new symmetries: quarks, leptons, and B-L," in 50 Years of Quarks, pp. 245-263, World Scientific, 2015.

[9] R. N. Mohapatra, "Neutrino mass as a signal of $\mathrm{TeV}$ scale physics," Nuclear Physics B, vol. 908, pp. 423-435, 2016.

[10] J. W. F. Valle, "Status and implications of neutrino masses: a brief panorama," International Journal of Modern Physics A, vol. 30, no. 13, Article ID 1530034, 2015.

[11] O. G. Miranda and J. W. Valle, "Neutrino oscillations and the seesaw origin of neutrino mass," Nuclear Physics. B, vol. 908, pp. 436-455, 2016.

[12] P. Minkowski, " $\mu \rightarrow \mathrm{e} \gamma$ at a rate of one out of $10^{9}$ muon decays?" Physics Letters B, vol. 67, no. 4, pp. 421-428, 1977.

[13] T. Yanagida, "Workshop on unified theories," KEK Report 79$18,1979$.

[14] M. Gell-Mann, P. Ramond, and R. Slansky, Supergravity, North Holland, Amsterdam, The Netherlands, 1979.

[15] S. L. Glashow, 1979 Cargese Summer Institute on Quarks and Leptons, Plenum, New York, NY, USA, 1980.

[16] R. N. Mohapatra and G. Senjanović, "Neutrino mass and spontaneous parity nonconservation," Physical Review Letters, vol. 44, no. 14, pp. 912-915, 1980.

[17] J. Schechter and J. W. F. Valle, "Neutrino masses in $\mathrm{SU}(2) \otimes \mathrm{U}(1)$ theories," Physical Review D, vol. 22, no. 9, pp. 2227-2235, 1980.

[18] M. Magg and C. Wetterich, "Neutrino mass problem and gauge hierarchy," Physics Letters B, vol. 94, no. 1, pp. 61-64, 1980.
[19] T. P. Cheng and L.-F. Li, "Neutrino masses, mixings, and oscillations in $\mathrm{SU}(2) \times \mathrm{U}(1)$ models of electroweak interactions," Physical Review D, vol. 22, no. 11, pp. 2860-2868, 1980.

[20] G. Lazarides, Q. Shafi, and C. Wetterich, "Proton lifetime and fermion masses in an SO(10) model," Nuclear Physics, Section B, vol. 181, no. 2, pp. 287-300, 1981.

[21] R. N. Mohapatra and G. Senjanović, "Neutrino masses and mixings in gauge models with spontaneous parity violation," Physical Review D, vol. 23, no. 1, pp. 165-180, 1981.

[22] J. Schechter and J. W. F. Valle, "Neutrinoless double- decay in $\mathrm{SU}(2) \times \mathrm{U}(1)$ theories," Physical Review D, vol. 25, no. 11, pp. 2951-2954, 1982.

[23] J. Schechter and J. W. F. Valle, "Neutrino decay and spontaneous violation of lepton number," Physical Review D, vol. 25, no. 3, pp. 774-783, 1982.

[24] R. N. Mohapatra and J. W. F. Valle, "Neutrino mass and baryon-number nonconservation in superstring models," Physical Review D, vol. 34, no. 5, pp. 1642-1645, 1986.

[25] R. N. Mohapatra, "Mechanism for understanding small neutrino mass in superstring theories," Physical Review Letters, vol. 56, no. 6, pp. 561-563, 1986.

[26] R. N. Mohapatra, "Status of neutrino mass in supersymmetric and superstring theories," in Proceedings of the International Symposium on Nuclear Beta Decays and Neutrino, pp. 42-52, Osaka, Japan, June 1986.

[27] R. N. Mohapatra, "Small neutrino masses in gauge theories," in Neutrino Physics, H. V. Klapdor and B. Povh, Eds., pp. 27-43, Springer, Heidelberg, Germany, 1988.

[28] C. H. Albright, "Search for a solution of the superstring neutrino mass problem without a high intermediate energy scale," Physics Letters B, vol. 178, no. 2-3, pp. 219-225, 1986.

[29] E. Witten, "New issues in manifolds of SU(3) holonomy," Nuclear Physics B, vol. 268, no. 1, pp. 79-112, 1986.

[30] S. Nandi and U. Sarkar, "Solution to the neutrino-mass problem in superstring E6 theory," Physical Review Letters, vol. 56, no. 6, pp. 564-567, 1986.

[31] J. W. F. Valle, "Gauge theories and the physics of neutrino mass," Progress in Particle and Nuclear Physics, vol. 26, pp. 91-171, 1991.

[32] D. Wyler and L. Wolfenstein, "Massless neutrinos in left-hand symmetric models," Nuclear Physics B, vol. 218, no. 1, pp. 205214,1983

[33] J. Bernabéu, A. Santamaria, J. Vidal, A. Mendez, and J. W. F. Valle, "Lepton flavour non-conservation at high energies in a superstring inspired standard model," Physics Letters B, vol. 187, no. 3-4, pp. 303-308, 1987.

[34] E. Ma, "Lepton-number nonconservation in E6 superstring models," Physics Letters B, vol. 191, no. 3, pp. 287-289, 1987.

[35] R. Foot, H. Lew, X.-G. He, and G. C. Joshi, "See-saw neutrino masses induced by a triplet of leptons," Zeitschrift für Physik C Particles and Fields, vol. 44, no. 3, pp. 441-444, 1989.

[36] W. Grimus and L. Lavoura, "The seesaw mechanism at arbitrary order: disentangling the small scale from the large scale," Journal of High Energy Physics, vol. 2000, no. 11, article no. 042, 2000.

[37] K. S. Babu, J. C. Pati, and F. Wilczek, "Fermion masses, neutrino oscillations, and proton decay in the light of superKamiokande," Nuclear Physics B, vol. 566, no. 1-2, pp. 33-91, 2000.

[38] K. S. Babu, J. C. Pati, and Z. Tavartkiladze, "Constraining proton lifetime in $\mathrm{SO}(10)$ with stabilized doublet-triplet splitting," Journal of High Energy Physics, vol. 2010, article 84, 2010. 
[39] S. M. Barr, "A different seesaw formula for neutrino masses," Physical Review Letters, vol. 92, no. 10, Article ID 101601, 2004.

[40] S. M. Barr and B. Kyae, "General analysis of corrections to the standard seesaw formula in grand unified models," Physical Review D, vol. 70, no. 7, Article ID 075005, 2004.

[41] C. H. Albright and S. M. Barr, "Resonant leptogenesis in a predictive SO(10) grand unified model," Physical Review D, vol. 70, no. 3, Article ID 033013, 2004.

[42] M. Malinský, J. C. Romão, and J. W. Valle, "Supersymmetric SO(10) seesaw mechanism with low B-L scale," Physical Review Letters, vol. 95, no. 16, Article ID 161801, 2005.

[43] M. Lindner, M. A. Schmidt, and A. Y. Smirnov, "Screening of Dirac flavor structure in the seesaw and neutrino mixing," Journal of High Energy Physics, vol. 2005, no. 7, article no. 048, 2005.

[44] T. Fukuyama, T. Kikuchi, and T. Osaka, "Non-thermal leptogenesis and a prediction of inflaton mass in supersymmetric $S O(10)$ model," Journal of Cosmology and Astroparticle Physics, vol. 06, article 005, 2005.

[45] S. K. Kang and C. S. Kim, "Extended double seesaw model for neutrino mass spectrum and low scale leptogenesis," Physics Letters B, vol. 646, no. 5-6, pp. 248-252, 2007.

[46] J. Ellis, D. V. Nanopoulos, and K. Olive, "Flipped heavy neutrinos. From the solar neutrino problem to baryogenesis," Physics Letters B, vol. 300, no. 1-2, pp. 121-127, 1993.

[47] S. K. Majee, M. K. Parida, and A. Raychaudhuri, "Neutrino mass and low-scale leptogenesis in a testable SUSY SO(10) model," Physics Letters, Section B: Nuclear, Elementary Particle and HighEnergy Physics, vol. 668, no. 4, pp. 299-302, 2008.

[48] M. Mitra, G. Senjanović, and F. Vissani, "Neutrinoless double beta decay and heavy sterile neutrinos," Nuclear Physics B, vol. 856, no. 1, pp. 26-73, 2012.

[49] J. C. Pati and A. Salam, "Unified Lepton-Hadron symmetry and a Gauge theory of the basic interactions," Physical Review D, vol. 8, no. 4, article 1240, 1973.

[50] J. C. Pati and A. Salam, "Lepton number as the fourth 'color," Physical Review D, vol. 10, no. 1, article 275, 1974.

[51] R. N. Mohapatra and J. C. Pati, "“Natural” left-right symmetry," Physical Review D, vol. 11, no. 9, pp. 2558-2561, 1975.

[52] G. Senjanovic and R. N. Mohapatra, "Exact left-right symmetry and spontaneous violation of parity," Physical Review D, vol. 12, no. 5, pp. 1502-1505, 1975.

[53] H. Georgi, "Coral gables 1975," in Proceedings of the Theories and Experiments in High Energy Physics, pp. 329-339, New York, NY, USA, 1975.

[54] H. Fritzsch and P. Minkowski, "Unified interactions of leptons and hadrons," Annals of Physics, vol. 93, no. 1-2, pp. 193-266, 1975.

[55] D. Chang, R. N. Mohapatra, and M. K. Parida, "Decoupling of parity- and SU(2)R-breaking scales: a new approach to left-right symmetric models," Physical Review Letters, vol. 52, no. 13, pp. 1072-1075, 1984.

[56] D. Chang, R. N. Mohapatra, and M. K. Parida, "New approach to left-right-symmetry breaking in unified gauge theories," Physical Review D, vol. 30, no. 5, article 1052, 1984.

[57] D. Chang, R. N. Mohapatra, J. M. Gipson, R. E. Marshak, and M. K. Parida, "Experimental tests of new $\mathrm{SO}(10)$ grand unification," Physical Review D, vol. 31, no. 7, pp. 1718-1732, 1985.

[58] R. N. Mohapatra and M. K. Parida, "Threshold effects on the mass-scale predictions in $\mathrm{SO}(10)$ models and solar-neutrino puzzle," Physical Review D, vol. 47, no. 1, pp. 264-272, 1993.
[59] B.-H. Lee and H. Min, "Quantum aspects of supersymmetric Maxwell Chern-Simons solitons," Physical Review. D. Third Series, vol. 51, no. 8, pp. 4458-4473, 1995.

[60] M. K. Parida, "Intermediate left-right gauge symmetry, unification of couplings and fermion masses in SUSY SO(10)XS(4)," Physical Review D, vol. 78, no. 5, Article ID 053004, 2008.

[61] M. Kadastic, K. Kanike, and M. Raidal, "Dark matter as the signal of grand unification," Physical Review D, vol. 80, no. 8, Article ID 085020, 2009, Erratum: Physical Review D, vol. 81, Article ID 029903, 2010.

[62] M. Kadastik, K. Kannike, and M. Raidal, "Matter parity as the origin of scalar dark matter," Physical Review D, vol. 81, no. 1, Article ID 015002, 2010.

[63] T. Hambye, "On the stability of particle dark matter," https:// arxiv.org/abs/1012.4587.

[64] M. Frigerio and T. Hambye, "Dark matter stability and unification without supersymmetry," Physical Review D, vol. 81, no. 7, Article ID 075002, 2010.

[65] Y. Mambrini, M. Nagata, K. Olive, J. Quevillon, and J. Zhang, "Dark matter and gauge coupling unification in nonsupersymmetric SO(10) grand unified models," Physical Review D, vol. 91, no. 9, Article ID 095010, 2015.

[66] C. Hagedorn, T. Ohlsson, S. Riad, and M. A. Schmidt, "Unification of gauge couplings in radiative neutrino mass models," Journal of High Energy Physics, vol. 2016, article 111, 2016.

[67] M. K. Parida, P. K. Sahu, and K. Bora, "Flavor unification, dark matter, proton decay, and other observable predictions with low-scale $\mathrm{S}_{4}$ symmetry," Physical Review D, vol. 83, no. 9, Article ID 093004, 2011.

[68] M. K. Parida, "Radiative seesaw in $\mathrm{SO}(10)$ with dark matter," Physics Letters B, vol. 704, no. 3, pp. 206-210, 2011.

[69] M. K. Parida, "Radiative see-saw formula in nonsupersymmetric SO(10) with dark matter," Pramana, vol. 79, no. 5, pp. 12711274, 2012.

[70] X. Chu and A. Yu Smirnov, "Neutrino mixing and masses in SO(10) GUTs with hidden sector and flavor symmetries," Journal of High Energy Physics, vol. 2016, article 135, 2016.

[71] G. L. Fogli, E. Lisi, A. Marrone, A. Palazzo, and A. M. Rotunno, "Global analysis of neutrino masses, mixings and phases: entering the era of leptonic CP violation searches," Physical Review D, vol. 86, no. 1, Article ID 013012, 2012.

[72] T. Schwetz, M. Tórtola, and J. W. Valle, "Where we are on $\theta_{13}$ : addendum to 'Global neutrino data and recent reactor fluxes: status of three-flavor oscillation parameters," New Journal of Physics, vol. 13, no. 10, p. 109401, 2011.

[73] D. V. Forero, M. Tartola, and J. W. F. Valle, "Neutrino oscillations refitted," Physical Review D, vol. 90, no. 9, Article ID 093006, 2014.

[74] M. C. Gonzalez-Garcia, M. Maltoni, and T. Schwetz, "Status of the CPT violating interpretations of the LSND signal," Physical Review D, vol. 68, no. 5, Article ID 053007, 2003.

[75] A. Aranda, C. Bonilla, S. Morisi, E. Peinado, and J. Valle, "Dirac neutrinos from flavor symmetry," Physical Review D, vol. 89, no. 3, Article ID 033001, 2014.

[76] J. W. Valle and C. Vaquera-Araujo, "Dynamical seesaw mechanism for Dirac neutrinos," Physics Letters B, vol. 755, pp. 363366, 2016.

[77] C. Bonilla and J. W. F. Valle, "Naturally light neutrinos in Diracon model," Physics Letters B, vol. 762, pp. 162-165, 2016.

[78] J. Gluza, T. Jelinski, and R. Szafron, "Lepton Number Violation and 'Diracness' of massive neutrinos composed of Majorana states," Physical Review D, vol. 93, no. 11, Article ID 113017, 2016. 
[79] H. V. Klapdor-Kleingrothaus, A. Dietz, L. Baudis et al., "Latest results from the HEIDELBERG-MOSCOW double beta decay experiment," The European Physical Journal A, vol. 12, no. 2, pp. 147-154, 2001.

[80] C. Arnaboldi, D. R. Artusa, F. T. Avignone et al., "Results from a search for the $0 \nu \beta \beta$-decay of ${ }^{130} \mathrm{Te}$, , Physical Review $C$, vol. 78, no. 3, Article ID 035502, 2008.

[81] C. E. Aalseth, F. T. Avignone III, R. L. Brodzinski et al., "IGEX ${ }^{76} \mathrm{Ge}$ neutrinoless double-beta decay experiment: prospects for next generation experiments," Physical Review D, vol. 65, no. 9, Article ID 092007, 2002.

[82] J. Argyriades, C. Augier, M. Bongrand et al., "Measurement of the double- $\beta$ decay half-life of ${ }^{150} \mathrm{Nd}$ and search for neutrinoless decay modes with the NEMO-3 detector," Physical Review $C$, vol. 80, no. 3, Article ID 032501, 2009.

[83] I. Abt, M. F. Altmann, A. Bakalyarov et al., "A new 76Ge double beta decay experiment at LNGS," https://arxiv.org/abs/hep-ex/ 0404039.

[84] A. K. Alfonso, D. R. Artusa, F. T. Avignone III et al., "Search for neutrinoless double-beta decay of ${ }^{130} \mathrm{Te}$ with CUORE-0," Physical Review Letters, vol. 115, no. 10, Article ID 102502, 7 pages, 2015.

[85] J. Adam, X. Bai, A. M. Baldini et al., "New limit on the leptonflavor-violating decay $\mu^{+} \rightarrow e^{+} \gamma$," Physical Review Letters, vol. 107, no. 17, Article ID 171801, 2011.

[86] K. Hayasaka, K. Abe, I. Adachi et al., "New search for $\tau \rightarrow \mu \gamma$ and $\tau \rightarrow e \gamma$ decays at Belle," Physics Letters B, vol. 666, no. 1, pp. 16-22, 2008.

[87] U. Bellgardt, G. Otter, R. Eichler et al., "Search for the decay $\mu^{+} \rightarrow e^{+} e^{+} e^{-}$, " Nuclear Physics B, vol. 299, no. 1, pp. 1-6, 1988.

[88] W. H. Bertl, R. Engfer, E. A. Hermes et al., "A search for $\mu-e$ conversion in muonic gold," The European Physical Journal C, vol. 47, no. 2, pp. 337-346, 2006.

[89] M. L. Brooks, Y. K. Chen, M. D. Cooper et al., "New limit for the lepton-family-number nonconserving decay $\mu^{+} \rightarrow e^{+} \gamma$," Physical Review Letters, vol. 83, pp. 1521-1524, 1999.

[90] B. Aubert, R. Barate, D. Boutigny et al., "Searches for Lepton flavor violation in the decays $\tau^{ \pm} \rightarrow e^{ \pm} \gamma$ and $\tau^{ \pm} \rightarrow \mu^{ \pm} \gamma$," Physical Review Letters, vol. 104, no. 2, Article ID 021802, 2010.

[91] Y. Kuno, "PRISM/PRIME," Nuclear Physics B - Proceedings Supplements, vol. 149, pp. 376-378, 2005.

[92] F. R. Joaquim and A. Rossi, "Phenomenology of the triplet seesaw mechanism with gauge and Yukawa mediation of SUSY breaking," Nuclear Physics B, vol. 765, no. 1-2, pp. 71-117, 2007.

[93] J. Adam, X. Bai, A. M. Baldini et al., "New constraint on the existence of the $\mu^{+} \rightarrow e^{+} \gamma$ decay," Physical Review Letters, vol. 110, no. 20, Article ID 201801, 2013.

[94] A. M. Baldini, Y. Bao, E. Baracchini et al., "Search for the lepton flavour violating decay $\mu^{+} \rightarrow e^{+} \gamma$ with the full dataset of the MEG experiment," The European Physical Journal C, vol. 76, no. 8, article 434, 2016.

[95] B. Aubert, Y. Karyotakis, J. P. Lees et al., "Searches for Lepton Flavor Violation in the Decays $\tau^{ \pm} \rightarrow e^{ \pm} \gamma$ and $\tau^{ \pm} \rightarrow \mu^{ \pm} \gamma$," Physical Review Letters, vol. 104, no. 2, Article ID 021802, 2010.

[96] R. H. Bernstein and P. S. Cooper, "Charged lepton flavor violation: an experimenter's guide," Physics Reports, vol. 532, no. 2, pp. 27-64, 2013.

[97] K. S. Babu, "Neutrino mixing pattern and its implications for charged lepton rare decays and CP violation," in Proceedings of the 5th Workshop on Neutrino Oscillations and their Origin (NOON '04), Tokyo, Japan, February 2004.
[98] S. T. Petcov, W. Rodejohann, T. Shindou, and Y. Takanishi, “The see-saw mechanism, neutrino Yukawa couplings, LFV decays $1_{i} \rightarrow 1_{j}+\gamma$ and leptogenesis," Nuclear Physics B, vol. 739, no. 1-2, pp. 208-233, 2006.

[99] S. Morisi and J. W. F. Valle, "Neutrino masses and mixing: a flavour symmetry roadmap," Fortschritte der Physik, vol. 61, no. 4-5, pp. 466-492, 2013.

[100] F. Deppisch, "Lepton flavour violation and flavour symmetries," Fortschritte der Physik, vol. 61, no. 4-5, pp. 622-644, 2013.

[101] A. de Gouvêa and P. Vogel, "Lepton flavor and number conservation, and physics beyond the standard model," Progress in Particle and Nuclear Physics, vol. 71, pp. 75-92, 2013.

[102] S. M. Boucenna, S. Morisi, and J. W. F. Valle, "The low-scale approach to neutrino masses," Advances in High Energy Physics, vol. 2014, Article ID 831598, 15 pages, 2014.

[103] J. Heeck, "Interpretation of lepton flavor violation," https:// arxiv.org/abs/1610.07623.

[104] Riazuddin, R. E. Marshak, and R. N. Mohapatra, "Majorana neutrinos and low-energy tests of electroweak models," Physical Review D, vol. 24, no. 5, pp. 1310-1317, 1981.

[105] S. Chatrchyan, V. Khachatryan, A. M. Sirunyan et al., "Search for heavy neutrinos and $W_{R}$ Bosons with right-handed couplings in a left-right symmetric model in $p p$ collisions at $\sqrt{s}=7 \mathrm{TeV}$," Physical Review Letters, vol. 109, no. 26, Article ID 261802, 2012.

[106] V. Khachatryan, A. M. Sirunyan, A. Tumasyan et al., "Search for heavy neutrinos and $\mathrm{W}$ bosons with right-handed couplings in proton-proton collisions at $\sqrt{s}=8 \mathrm{TeV}$,' The European Physical Journal C, vol. 74, article 3149, 2014.

[107] W. Keung and G. Senjanović, "Majorana neutrinos and the production of the right-handed charged gauge boson," Physical Review Letters, vol. 50, no. 19, pp. 1427-1430, 1983.

[108] C.-Y. Chen, P. S. Bhupal Dev, and R. N. Mohapatra, "Probing heavy-light neutrino mixing in left-right seesaw models at the LHC," Physical Review D, vol. 88, no. 3, Article ID 033014, 2013.

[109] B. Sahoo and M. K. Parida, "Low mass right-handed gauge bosons from minimal grand unified theories," Nuclear and Particle Physics Proceedings, vol. 273-275, pp. 2642-2644, 2016.

[110] M. K. Parida and B. Sahoo, "Planck-scale induced left-right gauge theory at LHC and experimental tests," Nuclear Physics. $B$, vol. 906, pp. 77-104, 2016.

[111] P. S. Bhupal Dev and R. N. Mohapatra, "Unified Explanation of the eejj, Diboson, and Dijet Resonances at the LHC," Physical Review Letters, vol. 115, no. 18, Article ID 181803, 2015.

[112] B. P. Nayak and M. K. Parida, "New mechanism for Type-II seesaw dominance in $\mathrm{SO}(10)$ with low-mass $\mathrm{ZI}, \mathrm{RH}$ neutrinos, and verifiable LFV, LNV and proton decay," European Physical Journal C, vol. 75, article no. 183, 2015.

[113] B. P. Nayak and M. K. Parida, "Dilepton events with displaced vertices, double beta decay, and resonant leptogenesis with type-II seesaw dominance, TeV scale ZI and heavy neutrinos," https://arxiv.org/abs/1509.06192.

[114] S. N. Gninenko and D. S. Gorbunov, "Search for GeV-scale sterile neutrinos responsible for active neutrino oscillations and baryon asymmetry of the universe," Advances in High Energy Physics, vol. 2012, Article ID 718259, 17 pages, 2012.

[115] L. Canetti, M. Drewes, and M. Shaposhnikov, "Sterile neutrinos as the origin of dark and baryonic matter," Physical Review Letters, vol. 110, no. 6, 2013.

[116] L. Canetti, M. Drewes, T. Frossard, and M. Shaposhnikov, "Dark matter, baryogenesis and neutrino oscillations from righthanded neutrinos," Physical Review D, vol. 87, no. 9, Article ID 093006, 2013. 
[117] J. C. Helo, S. G. Kovalenko, and M. Hirsch, "Heavy neutrino searches at the LHC with displaced vertices," Physical Review D - Particles, Fields, Gravitation and Cosmology, vol. 89, no. 7, Article ID 073005, 2014.

[118] S. Antusch, E. Cazzato, and O. Fischer, "Displaced vertex searches for sterile neutrinos at future lepton colliders," Journal of High Energy Physics, vol. 2016, article 7, 2016.

[119] B. P. Nayak and M. K. Parida, "Dilepton events with displaced vertices, double beta decay, and type-II seesaw dominance, $\mathrm{TeV}$ scale $Z^{\prime}$ and heavy neutrinos," https://arxiv.org/abs/1509.06192.

[120] S. Antusch, J. Baumann, and E. Fernández-Martínez, "Nonstandard neutrino interactions with matter from physics beyond the Standard Model," Nuclear Physics B, vol. 810, no. 1-2, pp. 369-388, 2009.

[121] S. Antusch, M. Blennow, E. Fernandez-Martinez, and J. LópezPavón, "Probing nonunitary mixing and $C P$ violation at a neutrino factory," Physical Review D, vol. 80, no. 3, Article ID 033002, 2009.

[122] S. Antush, C. Biggio, E. Fernandez-Martinez, M. Belen Gavela, and J. Lopez-Pavon, "Unitarity of the leptonic mixing matrix," Journal of High Energy Physics, vol. 2006, no. 10, p. 84, 2006.

[123] B. Dutta and R. N. Mohapatra, "Lepton flavor violation and neutrino mixings in a $3 \times 2$ seesaw model," Physical Review D, vol. 68, no. 5, Article ID 056006, 2003.

[124] A. Pilaftsis and T. E. J. Underwood, "Resonant leptogenesis," Nuclear Physics B, vol. 692, no. 3, pp. 303-345, 2004.

[125] J. Kersten and A. Y. Smirnov, "Right-handed neutrinos at CERN LHC and the mechanism of neutrino mass generation," Physical Review D, vol. 76, no. 7, Article ID 073005, 2007.

[126] C.-H. Lee, P. S. B. Dev, and R. N. Mohapatra, "Natural TeV-scale left-right seesaw mechanism for neutrinos and experimental tests," Physical Review D - Particles, Fields, Gravitation and Cosmology, vol. 88, no. 9, Article ID 093010, 2013.

[127] C. H. Albright and S. M. Barr, "Leptogenesis in the type III seesaw mechanism," Physical Review D, vol. 69, no. 7, Article ID 073010, 2004.

[128] C. H. Albright and S. Barr, "Predicting quark and lepton masses and mixings," Physics Letters B, vol. 452, no. 3-4, pp. 287-293, 1999.

[129] C. H. Albright, K. S. Babu, and S. M. Barr, "Minimality condition and atmospheric neutrino oscillations," Physical Review Letters, vol. 81, no. 6, pp. 1167-1170, 1998.

[130] P. Gu, M. Hirsch, U. Sarkar, and J. W. Valle, "Neutrino masses, leptogenesis, and dark matter in a hybrid seesaw model," Physical Review D, vol. 79, no. 3, Article ID 033010, 2009.

[131] S. Goswami, S. Khan, and A. Watanabe, "Hybrid textures in minimal seesaw mass matrices," Physics Letters B, vol. 693, no. 3, pp. 249-254, 2010.

[132] S. Chen, M. Frigerio, and E. Ma, "Hybrid seesaw neutrino masses with family symmetry," Nuclear Physics B, vol. 724, no. 1-2, pp. 423-431, 2005.

[133] P.-H. Gu and U. Sarkar, "Leptogenesis with linear, inverse or double seesaw," Physics Letters B, vol. 694, no. 3, pp. 226-232, 2010.

[134] M. Hirsch, S. Morisi, and J. W. F. Valle, "A4-based tri-bimaximal mixing within inverse and linear seesaw schemes," Physics Letters B, vol. 679, no. 5, pp. 454-459, 2009.

[135] J. Chakrabortty, A. S. Joshipura, P. Mehta, and S. K. Vempati, "Quasidegenerate neutrinos and maximal mixing in hybrid seesaw," in Proceedings of the 24th International Symposium on
Lepton-Photon Interactions at High Energies (LP09), T. Behnke and J. Mnich, Eds., vol. 493, Hamburg, Germany, August 2009.

[136] F. P. Deppisch and W.-C. Huang, "Dark matter and lepton flavour violation in a hybrid neutrino mass model," Journal of High Energy Physics, vol. 2015, article 66, 2015.

[137] B. Adhikary, B. Brahmachari, A. Ghosal, E. Ma, and M. K. Parida, "A4 symmetry and prediction of Ue3 in a modified Altarelli-Feruglio model," Physics Letters B, vol. 638, no. 4, pp. 345-349, 2006.

[138] B. Adhikary, M. Chakraborty, and A. Ghosal, "Scaling ansatz, four zero Yukawa textures, and large $\theta 13$," Physical Review D, vol. 86, no. 1, Article ID 013015, 2012.

[139] B. Adhikary, A. Ghosal, and P. Roy, " $\theta 13, \mu \tau$ symmetry breaking,and neutrino Yukawa textures," International Journal of Modern Physics A, vol. 28, no. 24, Article ID 1350118, 2013.

[140] M. Chakraborty, H. Zeen Devi, and A. Ghosal, "Scaling ansatz with texture zeros in linear seesaw," Physics Letters B, vol. 741, pp. 210-216, 2015.

[141] A. Ghosal and R. Samanta, "Probing texture zeros with scaling ansatz in inverse seesaw," Journal of High Energy Physics, vol. 2015, article no. 77, 2015.

[142] R. Samanta and A. Ghosal, "Probing maximal zero textures with broken cyclic symmetry in inverse seesaw," Nuclear Physics B, vol. 911, pp. 846-862, 2016.

[143] R. Sinha, R. Samanta, and A. Ghosal, "Maximal zero textures in Linear and Inverse seesaw," Physics Letters B, vol. 759, pp. 206213, 2016.

[144] S. Pramanick and A. Raychaudhuri, "A4 -based seesaw model for realistic neutrino masses and mixing," Physical Review D, vol. 93, no. 3, Article ID 033007, 2016.

[145] S. Pramanick and A. Raychaudhuri, "Neutrino mass model with $S_{3}$ symmetry and seesaw interplay," Physical Review D, vol. 94, no. 11, 2016.

[146] M. K. Parida, B. P. Nayak, R. Satpathy, and R. L. Awasthi, "Standard coupling unification in SO (10), hybrid seesaw neutrino mass and leptogenesis, dark matter, and proton lifetime predictions," https://arxiv.org/abs/1608.03956.

[147] B. Bajc, G. Senjanović, and F. Vissani, "b- $\tau$ unification and large atmospheric mixing: a case for a noncanonical seesaw mechanism," Physical Review Letters, vol. 90, no. 5, Article ID 051802, 2003.

[148] H. Goh, R. Mohapatra, and S. Ng, "Minimal SUSY SO(10), b- $\tau$ unification and large neutrino mixings," Physics Letters B, vol. 570, no. 3-4, pp. 215-221, 2003.

[149] H. S. Goh, R. N. Mohapatra, and S. Ng, "Minimal supersymmetric $\mathrm{SO}(10)$ model and predictions for neutrino mixings and leptonic CP violation," Physical Review D, vol. 68, no. 11, 2003.

[150] G. Altarelli and G. Blankenburg, "Different SO(10) paths to fermion masses and mixings," Journal of High Energy Physics, vol. 2011, no. 3, article no. 133, 2011.

[151] B. Bajc, G. Senjanovic, and F. Vissani, "How neutrino and charged fermion masses are connected within minimal supersymmetric SO(10)," https://arxiv.org/abs/hep-ph/0110310.

[152] S. Bertolini, M. Frigerio, and M. Malinský, "Fermion masses in supersymmetric $\mathrm{SO}(10)$ with type II seesaw mechanism: a nonminimal predictive scenario," Physical Review D, vol. 70, no. 9, Article ID 095002, 2004.

[153] P. S. Dev, R. N. Mohapatra, and M. Severson, "Neutrino mixings in $S O(10)$ with type II seesaw mechanism and $\theta_{13}$," Physical Review D, vol. 84, no. 5, 2011. 
[154] R. N. Mohapatra, M. K. Parida, and G. Rajasekaran, "High scale mixing unification and large neutrino mixing angles," Physical Review D, vol. 69, no. 5, Article ID 053007, 2004.

[155] R. N. Mohapatra, M. K. Parida, and G. Rajasekaran, “Threshold effects on quasidegenerate neutrinos with high-scale mixing unification," Physical Review D, vol. 71, no. 5, Article ID 057301, 2005.

[156] S. K. Agarwalla, M. K. Parida, R. N. Mohapatra, and G. Rajasekaran, "Neutrino mixings and leptonic," Physical Review $D$, vol. 75 , no. 3, 2007.

[157] H. S. Goh, R. N. Mohapatra, and S. Nasri, "SO(10) symmetry breaking and type II seesaw formula," Physical Review D, vol. 70, no. 7, Article ID 075022, 2004.

[158] B. Dutta, Y. Mimura, and R. N. Mohapatra, "Origin of quarklepton flavor in $\mathrm{SO}(10)$ with type II seesaw mechanism," Physical Review D, vol. 80, no. 9, Article ID 095021, 2009.

[159] S.-L. Chen, R. N. Mohapatra, S. Nussinov, and Y. Zhang, "Rparity breaking via type II seesaw, decaying gravitino dark matter and PAMELA positron excess," Physics Letters B, vol. 677, no. 5, pp. 311-317, 2009.

[160] R. N. Mohapatra and M. K. Parida, “Type-II seesaw dominance in nonsupersymmetric and split-supersymmetry $\mathrm{SO}(10)$ models and proton lifetime," Physical Review D, vol. 84, no. 9, Article ID 095021, 2011.

[161] A. Melfo, M. Nemevšek, F. Nesti, G. Senjanović, and Y. Zhang, "Type II neutrino seesaw mechanism at the LHC: the roadmap," Physical Review D, vol. 85, no. 5, Article ID 055018, 2012.

[162] G. Bambhaniya, P. S. Bhupal Dev, and S. Goswami, "The scalar triplet contribution to lepton flavour violation and neutrinoless double beta decay in Left-Right Symmetric Model," Journal of High Energy Physics, vol. 2016, article 46, 2016.

[163] G. 't Hooft et al., "Naturalness, chiral symmetry breaking and spontaneous chiral symmetry breaking," in Proceedings of the 1979 Cargese Summer Institute on Recent Developments in Gauge Theories, G. 't Hooft, C. Itzykson, A. Jaffe et al., Eds., Plenum Press, New York, NY, USA, 1980.

[164] P. S. Bhupal Dev and R. N. Mohapatra, "TeV scale inverse seesaw model in $\mathrm{SO}(10)$ and leptonic nonunitarity effects," Physical Review D, vol. 81, no. 1, Article ID 013001, 2010.

[165] P. S. Bhupal Dev and R. N. Mohapatra, "Electroweak symmetry breaking and proton decay in $S O(10)$ supersymmetric GUT with TeV $W_{R}$," Physical Review D, vol. 82, no. 3, Article ID 035014, 2010.

[166] R. L. Awasthi and M. K. Parida, "Inverse seesaw mechanism in nonsupersymmetric $\mathrm{SO}(10)$, proton lifetime, nonunitarity effects, and a low-mass $Z^{\prime}$ boson," Physical Review D, vol. 86, no. 9, Article ID 093004, 2012.

[167] A. Das and N. Okada, "Inverse seesaw neutrino signatures at the LHC and ILC," Physical Review D, vol. 88, no. 11, Article ID 113001, 2013.

[168] Y. Ahn, S. K. Kang, and C. S. Kim, "A model for pseudoDirac neutrinos: leptogenesis and ultra-high energy neutrinos," Journal of High Energy Physics, vol. 2016, article 92, 2016.

[169] H. S. Cheon, S. K. Kang, and C. S. Kim, "Low scale leptogenesis and dark matter candidates in an extended seesaw model," Journal of Cosmology and Astroparticle Physics, vol. 2008, no. 5, article 004, 2008.

[170] H. S. Cheon, S. K. Kang, and C. S. Kim, "Doubly coexisting dark matter candidates in an extended seesaw model," Physics Letters B, vol. 675, no. 2, pp. 203-209, 2009.
[171] S. K. Kang and A. Patra, "keV sterile neutrino dark matter and low scale leptogenesis," https://arxiv.org/abs/1412.4899.

[172] S. K. Majee, M. K. Parida, and A. Raychaudhuri, "Neutrino mass and low-scale leptogenesis in a testable SUSY SO(10) model," Physics Letters B, vol. 668, no. 4, pp. 299-302, 2008.

[173] M. K. Parida and A. Raychaudhuri, "Inverse seesaw mechanism, leptogenesis, observable proton decay, and $\triangle_{R}^{ \pm \pm}$in supersymmetric $S O(10)$ with heavy $W_{R}$, Physical Review D, vol. 82, no. 9, Article ID 093017, 2010.

[174] S. K. Majee, M. K. Parida, A. Raychaudhuri, and U. Sarkar, "Low intermediate scales for leptogenesis in supersymmetric $\mathrm{SO}(10)$ grand unified theories," Physical Review D, vol. 75, no. 7, Article ID 075003, 2007.

[175] M. K. Parida and S. Patra, "Left-right models with light neutrino mass prediction and dominant neutrinoless double beta decay rate," Physics Letters B, vol. 718, no. 4-5, pp. 1407-1412, 2013.

[176] R. L. Awasthi, M. K. Parida, and S. Patra, "Neutrino masses, dominant neutrinoless double beta decay, and observable lepton flavor violation in left-right models and $\mathrm{SO}(10)$ grand unification with low mass $W_{R}, Z_{R}$ bosons," Journal of High Energy Physics, vol. 2013, no. 8, article 122, 2013.

[177] M. K. Parida, R. L. Awasthi, and P. K. Sahu, "Proton decay and new contributions to $0 \nu 2 \beta$ decay in $\mathrm{SO}(10)$ with low-mass $Z^{\prime}$ boson, observable $n-\bar{n}$ oscillation, lepton flavor violation, and rare kaon decay," Journal of High Energy Physics, vol. 2015, article 45, 2015.

[178] E. Akhmedov, M. Lindner, E. Schnapka, and J. W. F. Valle, "Dynamical left-right symmetry breaking," Physical Review D, vol. 53, no. 5, pp. 2752-2780, 1996.

[179] F. F. Deppisch, "Neutrinos and LHC physics," Acta Physica Polonica B, vol. 46, no. 11, pp. 2301-2310, 2015.

[180] F. F. Deppisch, P. S. Bhupal Dev, and A. Pilaftsis, "Neutrinos and collider physics," New Journal of Physics, vol. 17, no. 3, Article ID 036005, 2015.

[181] A. S. Joshipura and J. W. F. Valle, "Invisible Higgs decays and neutrino physics," Nuclear Physics, Section B, vol. 397, no. 1-2, pp. 105-122, 1993.

[182] M. A. Díaz, M. A. García-Jareño, D. A. Restrepo, and J. W. F. Valle, "Seesaw majoron model of neutrino mass and novel signals in Higgs boson production at LEP," Nuclear Physics B, vol. 527, no. 1-2, pp. 44-60, 1998.

[183] C. Bonilla, J. W. F. Valle, and J. C. Romao, "Neutrino mass and invisible Higgs decays at the LHC," Physical Review D, vol. 91, no. 11, Article ID 113015, 2015.

[184] F. Deppisch and J. W. F. Valle, "Enhanced lepton flavor violation in the supersymmetric inverse seesaw model," Physical Review D, vol. 72, no. 3, Article ID 036001, 2005.

[185] F. Deppisch, T. S. Kosmas, and J. W. F. Valle, "Enhanced $\mu$ e- conversion in nuclei in the inverse seesaw model," Nuclear Physics B, vol. 752, no. 1-2, pp. 80-92, 2006.

[186] F. F. Deppisch, N. Desai, and J. W. F. Valle, "Is charged lepton flavor violation a high energy phenomenon?" Physical Review D, vol. 89, no. 5, Article ID 051302, 2014.

[187] A. Abada, M. E. Krauss, W. Porod, F. Staub, A. Vicente, and C. Weiland, "Lepton flavor violation in low-scale seesaw models: SUSY and non-SUSY contributions," Journal of High Energy Physics, vol. 2014, no. 11, article no. 48, 2014.

[188] S. M. Boucenna, J. W. Valle, and A. Vicente, "Predicting charged lepton flavor violation from 3-3-1 gauge symmetry," Physical Review D, vol. 92, no. 5, Article ID 053001, 2015. 
[189] V. Cirigliano, A. Kurylov, M. J. Ramsey-Musolf, and P. Vogel, "Neutrinoless double beta decay and lepton flavor violation," Physical Review Letters, vol. 93, no. 23, Article ID 231802, 2004.

[190] M. E. Peskin and T. Takeuchi, "New constraint on a strongly interacting Higgs sector," Physical Review Letters, vol. 65, no. 8, pp. 964-967, 1990.

[191] G. Altarelli and R. Barbieri, "Vacuum polarization effects of new physics on electroweak processes," Physics Letters B, vol. 253, no. 1-2, pp. 161-167, 1991.

[192] M. E. Peskin and T. Takeuchi, "Estimation of oblique electroweak corrections," Physical Review D, vol. 46, no. 1, pp. 381409, 1992.

[193] E. Kh. Akhmedov, A. S. Josipura, S. Ranfone, and J. W. F. Valle, "Left-right symmetry and neutrino stability," Nuclear Physics B, vol. 441, no. 1-2, pp. 61-75, 1995, (LRS model with additional spontaneously broken $\mathrm{U}(1)$ global symmetry: $v \tau \rightarrow$ $v+\mathrm{J}$ (Majoron) leading to nonvanishing mnu (due to S- $v$ mixing . Gives low WR scale. Charged LFV Majoron decays: Br. $(l i \rightarrow$ $l i+J)>10_{-4}-10_{-6}$.

[194] A. Kumar and R. N. Mohapatra, "A model for neutrino decays," Physics Letters B, vol. 150, no. 1-3, pp. 191-195, 1985.

[195] E. Akhmedov, M. Lindner, E. Schnapka, and J. W. F. Valle, "Leftright symmetry breaking in NJL approach," Physics Letters B, vol. 368, no. 4, pp. 270-280, 1996.

[196] M. K. Parida and A. Raychaudhuri, "Low-mass parity restoration, weak-interaction phenomenology, and grand unification," Physical Review D, vol. 26, no. 9, pp. 2364-2377, 1982.

[197] M. Malinsky, J. C. Romao, and J. W. F. Valle, "Supersymmetric SO(10) Seesaw mechanism with low B-L scale," Physical Review Letters, vol. 95, no. 16, Article ID 161801, 2005.

[198] E. A. Garcés, O. G. Miranda, M. A. Tórtola, and J. W. Valle, "Low-energy neutrino-electron scattering as a standard model probe: the potential of LENA as case study," Physical Review D, vol. 85, no. 7, Article ID 073006, 2012.

[199] S. Blanchet, P. S. Bhupal Dev, and R. N. Mohapatra, "Leptogenesis with TeV-scale inverse seesaw model in SO(10)," Physical Review D, vol. 82, no. 11, Article ID 115025, 2010.

[200] A. Vicente, "Lepton flavor violation in SUSY left-right symmetric theories," Journal of Physics: Conference Series, vol. 259, no. 1, Article ID 012065, 2010.

[201] A. Abada and M. Lucente, "Looking for the minimal inverse seesaw realisation," Nuclear Physics B, vol. 885, pp. 651-678, 2014.

[202] C. Weiland, Effects of fermionic siglet neutrinos on high-and lowenergy observables [Ph.D. thesis], École Normale Supérieure de Lyon, Lyon, France, 2010.

[203] M. Lindner, M. Platscher, and F. Queiroz, "A call for new physics: the muon magnetic moment, and lepton flavor violation," https://arxiv.org/abs/1610.06587.

[204] C. Bonilla, M. E. Krauss, T. Opterkuch, and W. Porod, "Perspectives for detecting lepton flavour violation in left-right symmetric models," https://arxiv.org/abs/1611.07025.

[205] E. Ma and U. Sarkar, "Neutrino masses and leptogenesis with heavy higgs triplets," Physical Review Letters, vol. 80, no. 26, article 5716, 1998.

[206] E. Ma, "All-purpose neutrino mass matrix," Physical Review D, vol. 66, no. 11, Article ID 117301, 2002.

[207] F. F. Deppisch, P. S. B. Dev, and A. Pilaftsis, "Neutrinos and collider physics," New Journal of Physics, vol. 17, no. 7, Article ID 075019, 2015.
[208] J. W. F. Valle and M. Singer, "Lepton-number violation with quasi-Dirac neutrinos," Physical Review D, vol. 28, no. 3, pp. 540-545, 1983.

[209] J. W. F. Valle, "Neutrinoless double- $\beta$ decay with quasi-Dirac neutrinos," Physical Review D, vol. 27, no. 7, pp. 1672-1674, 1983.

[210] B. Brahmachari, U. Sarkar, and K. Sridhar, "Ruling out low energy left-right symmetry in unified theories," Physics Letters $B$, vol. 297, no. 1-2, pp. 105-110, 1992.

[211] F. F. Deppisch, L. Graf, S. Kulkarni et al., "Reconciling the $2 \mathrm{TeV}$ excesses at the LHC in a linear seesaw left-right model," Physical Review D, vol. 93, no. 1, Article ID 013011, 2016.

[212] F. F. Deppisch, T. E. Gonzalo, S. Patra, N. Sahu, and U. Sarkar, "Double beta decay, lepton flavor violation, and collider signatures of left-right symmetric models with spontaneous $D$-parity breaking," Physical Review D - Particles, Fields, Gravitation and Cosmology, vol. 91, Article ID 015018, 2015.

[213] F. F. Deppisch, T. E. Gonzalo, S. Patra, N. Sahu, and U. Sarkar, "Signal of right-handed charged gauge bosons at the LHC?" Physical Review D, vol. 90, no. 5, Article ID 053014, 2014.

[214] J. Barry, L. Dorame, and W. Rodejohann, "Linear collider test of a neutrinoless double beta decay mechanism in left-right symmetric theories," The European Physical Journal C, vol. 72, no. 5, article 2023, 2012.

[215] J. Barry and W. Rodejohann, "Lepton number and flavour violation in $\mathrm{TeV}$-scale left-right symmetric theories with large left-right mixing," Journal of High Energy Physics, vol. 2013, no. 9, article 153, 2013.

[216] B. A. Dobrescu and Z. Liu, "W' Boson near $2 \mathrm{TeV}$ : predictions for Run 2 of the LHC," Physical Review Letters, vol. 115, no. 21, Article ID 211802, 2015.

[217] S. P. Das, F. F. Deppisch, O. Kittel, and J. W. F. Valle, "Heavy neutrinos and lepton flavor violation in left-right symmetric models at the LHC," Physical Review D-Particles, Fields, Gravitation and Cosmology, vol. 86, no. 5, Article ID 055006, 2012.

[218] A. Ilakovac and A. Pilaftsis, "Flavour-violating charged lepton decays in seesaw-type models," Nuclear Physics B, vol. 437, no. 3, pp. 491-519, 1995.

[219] F. Deppisch and J. W. F. Valle, "Enhanced lepton flavor violation in the supersymmetric inverse seesaw model," Physical Review D, vol. 72, Article ID 036001, 2005.

[220] C. Arina, F. Bazzocchi, N. Fornengo, J. C. Romao, and J. W. Valle, "Minimal supergravity scalar neutrino dark matter and inverse seesaw neutrino masses," Physical Review Letters, vol. 101, no. 16, Article ID 161802, 2008.

[221] M. Malinský, T. Ohlsson, Z.-Z. Xing, and H. Zhang, "Nonunitary neutrino mixing and $\mathrm{CP}$ violation in the minimal inverse seesaw model," Physics Letters B, vol. 679, no. 3, pp. 242248, 2009.

[222] M. Hirsch, T. Kernreiter, J. C. Romão, and A. V. del Moral, "Minimal supersymmetric inverse seesaw: neutrino masses, lepton flavour violation and LHC phenomenology," Journal of High Energy Physics, vol. 2010, article no. 103, 2010.

[223] F. Deppisch, T. Kosmas, and J. Valle, "Enhanced $\mu^{-}-\mathrm{e}^{-}$conversion in nuclei in the inverse seesaw model," Nuclear Physics B, vol. 752, no. 1-2, pp. 80-92, 2006.

[224] E. Fernández-Martínez, M. Gavela, J. López-Pavón, and O. Yasuda, "CP-violation from non-unitary leptonic mixing," Physics Letters B, vol. 649, no. 5-6, pp. 427-435, 2007.

[225] K. Kanaya, "Neutrino mixing in the minimal SO(10) model," Progress of Theoretical Physics, vol. 64, no. 6, pp. 2278-2281, 1980. 
[226] M. Malinsky, T. Ohlsson, and H. Zhang, "Nonunitarity effects in a realistic low-scale seesaw model," Physical Review D, vol. 79, no. 7, Article ID 073009, 2009.

[227] G. Altarelli and D. Meloni, "CP violation in neutrino oscillations and new physics," Nuclear Physics B, vol. 809, no. 1-2, pp. 158182, 2009.

[228] F. del Aguila and J. A. Aguilar-Saavedra, "Electroweak scale seesaw and heavy Dirac neutrino signals at LHC," Physics Letters $B$, vol. 672, no. 2, pp. 158-165, 2009.

[229] F. del Aguila, J. A. Aguilar-Saavedra, and J. de Blas, "Trilepton signals: the golden channel for seesaw searches at LHC," Acta Physica Polonica B, vol. 40, pp. 2901-2911, 2009.

[230] A. Van Der Schaaf, "Muon physics at a neutrino factory," Journal of Physics G: Nuclear and Particle Physics, vol. 29, no. 12, pp. 2755-2762, 2003.

[231] Y. Kuno, "PRISM/PRIME," Nuclear Physics B, vol. 149, pp. 376378, 2005.

[232] C. Das and M. Parida, "New formulas and predictions for running fermion masses at higher scales in SM, 2HDM, and MSSM," The European Physical Journal C, vol. 20, no. 1, pp. 121137, 2001.

[233] K. S. Babu and R. N. Mohapatra, "Predictive neutrino spectrum in minimal SO(10) grand unification," Physical Review Letters, vol. 70 , no. 19, pp. 2845-2848, 1993.

[234] V. Tello, Connections between the high and low energy violation of lepton and flavor numbers in the minimal left-right symmetric model (submitted for the degree of Doctor of Philosophy) [Ph.D. thesis], SISSA, Trieste, Italy, 2012.

[235] M. Nemevsek, F. Nesti, G. Senjanovic, and V. Tello, "Neutrinoless double beta decay: low left-right symmetry scale?" https://arxiv.org/abs/1112.3061.

[236] F. F. Deppisch, M. Hirsch, and H. Päs, "Neutrinoless doublebeta decay and physics beyond the standard model," Journal of Physics G: Nuclear and Particle Physics, vol. 39, no. 12, Article ID 124007, 2012.

[237] R. N. Mohapatra, "Limits on the mass of the right-handed Majorana neutrino," Physical Review D, vol. 34, no. 3, pp. 909910, 1986.

[238] M. Hirsch, H. Klapdor-Kleingrothaus, and O. Panella, "Double beta decay in left-right symmetric models," Physics Letters B, vol. 374, no. 1-3, pp. 7-12, 1996.

[239] V. Tello, M. Nemevšek, F. Nesti, G. Senjanović, and F. Vissani, "Left-right symmetry: from the LHC to neutrinoless double beta decay," Physical Review Letters, vol. 106, no. 15, Article ID 151810, 2011.

[240] P. S. Bhupal Dev, S. Goswami, and M. Mitra, "TeV-scale leftright symmetry and large mixing effects in neutrinoless double beta decay," Physical Review D, vol. 91, no. 11, Article ID 113004, 2015.

[241] R. N. Mohapatra and J. D. Vergados, "New contribution to neutrinoless double beta decay in gauge models," Physical Review Letters, vol. 47, pp. 1713-1716, 1981.

[242] C. E. Picciotto and M. S. Zahir, "Neutrinoless double- $\beta$ decay in left-right-symmetric models," Physical Review D, vol. 26, pp. 2320-2325, 1982.

[243] M. Doi and T. Kotani, "Neutrinoless modes of double beta decay," Progress of Theoretical Physics, vol. 89, no. 1, pp. 139-159, 1993.

[244] K. Muto, I. Blender, and H. V. Klapdor-Kleingrothaus, "Effects of ground-state correlations on $2 \nu \beta \beta$ decay rates and limitations of the QRPA approach," Zeitschrift für Physik A, vol. 334, no. 2, pp. 177-186, 1989.
[245] M. Hirsch, K. Muto, T. Oda, and H. V. Klapdor-Kleingrothaus, "Nuclear structure calculation of $\beta^{+} \beta^{+}, \beta^{+} / \mathrm{EC}$ and EC/EC decay matrix elements," Zeitschrift für Physik A Hadrons and Nuclei, vol. 347, no. 3, pp. 151-160, 1994.

[246] J. J. Gomez-Cadenas, J. Martin-Albo, M. Mezzetto, F. Monrabal, and M. Sorel, "The search for neutrinoless double beta decay," La Rivista del Nuovo Cimento, vol. 35, no. 2, pp. 29-98, 2012.

[247] J. Lopez-Pavon, S. Pascoli, and C.-F. Wong, "Can heavy neutrinos dominate neutrinoless double beta decay?” Physical Review D, vol. 87, no. 9, Article ID 093007, 2013.

[248] G. Pantis, F. Šimkovic, J. D. Vergados, and A. Faessler, "Neutrinoless double beta decay within the quasiparticle randomphase approximation with proton-neutron pairing," Physical Review C, vol. 53, no. 2, pp. 695-707, 1996.

[249] K. Muto, E. Bender, and H. V. Klapdor, "Nuclear structure effects on the neutrinoless double beta decay," Zeitschrift für Physik A, vol. 334, no. 2, pp. 187-194, 1989.

[250] J. Suhonen and O. Civitarese, "Weak-interaction and nuclearstructure aspects of nuclear double beta decay," Physics Report, vol. 300, no. 3-4, pp. 123-214, 1998.

[251] J. Kotila and F. Iachello, "Phase-space factors for double- $\beta$ decay," Physical Review C, vol. 85, no. 3, Article ID 034316, 2012.

[252] F. Šimkovic, G. Pantis, J. D. Vergados, and A. Faessler, "Additional nucleon current contributions to neutrinoless double $\beta$ decay," Physical Review C, vol. 60, no. 5, Article ID 055502, 1999.

[253] A. Faessler, A. Meroni, S. T. Petcov, F. Šimkovic, and J. Vergados, "Uncovering multiple CP-nonconserving mechanisms of $(\beta \beta) 0 v$ decay," Physical Review D, vol. 83, no. 11, Article ID 113003, 2011.

[254] M. Fukugita and T. Yanagida, "Barygenesis without grand unification," Physics Letters B, vol. 174, no. 1, pp. 45-47, 1986.

[255] S. Davidson and A. Ibarra, "A lower bound on the right-handed neutrino mass from leptogenesis," Physics Letters B, vol. 535, no. 1-4, pp. 25-32, 2002.

[256] T. Hambye and G. Senjanović, "Consequences of triplet seesaw for leptogenesis," Physics Letters B, vol. 582, no. 1-2, pp. 73-81, 2004.

[257] M. Y. Khlopov and A. D. Linde, "Is it easy to save the gravitino?" Physics Letters B, vol. 138, no. 4, pp. 265-268, 1984.

[258] L. Boubekeur, T. Hambye, and G. Senjanović, "Low-scale leptogenesis and soft supersymmetry breaking," Physical Review Letters, vol. 93, no. 11, Article ID 111601, 2004.

[259] K. Babu, A. Bachri, and H. Aissaoui, "Leptogenesis in minimal left-right symmetric models," Nuclear Physics B, vol. 738, no. 1-2, pp. 76-92, 2006.

[260] X. Ji, Y. Li, R. Mohapatra, S. Nasri, and Y. Zhang, "Leptogenesis in realistic SO(10) models," Physics Letters B, vol. 651, no. 2-3, pp. 195-207, 2007.

[261] K. Kumekawa, T. Moroi, and T. Yanagida, "Flat potential for inflaton with a discrete $R$-invariance in supergravity," Progress of Theoretical Physics, vol. 92, no. 2, pp. 437-447, 1994.

[262] G. F. Giudice, A. Riotto, I. Tkachev, and M. Peloso, "Production of massive fermions at preheating and leptogenesis," Journal of High Energy Physics, vol. 1999, 1999.

[263] T. Asaka, K. Hamaguchi, M. Kawasaki, and T. Yanagida, "Leptogenesis in inflaton decay," Physics Letters B, vol. 464, no. 1-2, pp. 12-18, 1999.

[264] R. Jeannerot, S. Khalil, G. Lazarides, and Q. Shafi, "Inflation and monopoles in supersymmetric SU $(4) \mathrm{c} \times \mathrm{SU}(2) \mathrm{L} \times \mathrm{SU}(2) \mathrm{R}$," Journal of High Energy Physics, vol. 2000, no. 10, article no. 012, 2000 . 
[265] J. C. Pati, "Leptogenesis and neutrino oscillations within a predictive G(224)/SO(10) framework," Physical Review D, vol. 68, Article ID 072002, 2003.

[266] J. C. Pati, "Probing grand unification through neutrino oscillations, leptogenesis, and proton decay," International Journal of Modern Physics A, vol. 18, no. 22, pp. 4135-4156, 2003, Subnucl. Ser, vol. 40, pp. 194-236, 2003.

[267] E. Molinaro, CP violation in the lepton sector, thermal leptogenesis and lepton flavor violating processes [Ph.D. thesis], SISSA, Trieste, Italy, 2010.

[268] M. Hirsch, J. W. Valle, M. Malinský, J. C. Romão, and U. Sarkar, "Thermal leptogenesis in extended supersymmetric seesaw model," Physical Review D, vol. 75, no. 1, Article ID 011701, 2007.

[269] E. Ma, N. Sahu, and U. Sarkar, "Leptogenesis below the Davidson-Ibarra bound," Journal of Physics G: Nuclear and Particle Physics, vol. 32, no. 11, pp. L65-L68, 2006.

[270] P. Gu and U. Sarkar, "Leptogenesis with linear, inverse or double seesaw," Physics Letters B, vol. 694, no. 3, pp. 226-232, 2010.

[271] P.-H. Gu, M. Hirsch, U. Sarkar, and J. W. F. Valle, "Neutrino masses, leptogenesis, and dark matter in a hybrid seesaw model," Physical Review D, vol. 79, no. 3, Article ID 033010, 2009.

[272] E. Ma, N. Sahu, and U. Sarkar, "Low-energy thermal leptogenesis in an extended NMSSM model," Journal of Physics G: Nuclear and Particle Physics, vol. 34, no. 4, article 741, 2007.

[273] F. F. Deppisch, J. Harz, and M. Hirsch, "Falsifying high-scale leptogenesis at the LHC," Physical Review Letters, vol. 112, no. 22, Article ID 221601, 2014.

[274] S. Blanchet, T. Hambye, and F.-X. Josse-Michaux, "Reconciling leptogenesis with observable $\mu \rightarrow \mathrm{e} \gamma$ rates," Journal of High Energy Physics, vol. 2010, no. 4, article no. 023, 2010.

[275] F. F. Deppisch, J. Harz, W.-C. Huang, M. Hirsch, and H. Päs, "Falsifying high-scale baryogenesis with neutrinoless double beta decay and lepton flavor violation," Physical Review D, vol. 92, no. 3, Article ID 036005, 2015.

[276] A. Abada, G. Arcadi, and M. Lucente, "Dark matter in the minimal inverse seesaw mechanism," Journal of Cosmology and Astroparticle Physics, vol. 2014, no. 10, article 001, 2014.

[277] A. Abada and S. Nasri, "Renormalization group equations of a cold dark matter two-singlet model," Physical Review D, vol. 88, no. 1, Article ID 016006, 2013.

[278] H. Nishino, S. Clark, K. Abe et al., "Search for proton decay via $p \rightarrow e^{+} \pi^{0}$ and $p \rightarrow \mu^{+} \pi^{0}$ in a large water cherenkov detector," Physical Review Letters, vol. 102, no. 14, Article ID 141801, 2009.

[279] S. Raby, T. Walker, and K. S. Babu, "DUSEL theory white paper," https://arxiv.org/abs/0810.4551.

[280] M. Shiozawa, “The Hyper-Kamiokande prospect," in Proceedings of the 13th International Conference on Topics in Astroparticle and Underground Physics (TAUP '13), Asilomar, Calif, USA, September 2013.

[281] J. Bergström, M. Malinský, T. Ohlsson, and H. Zhang, "Renormalization group running of neutrino parameters in the inverse seesaw model," Physical Review D, vol. 81, no. 11, Article ID 116006, 2010. 

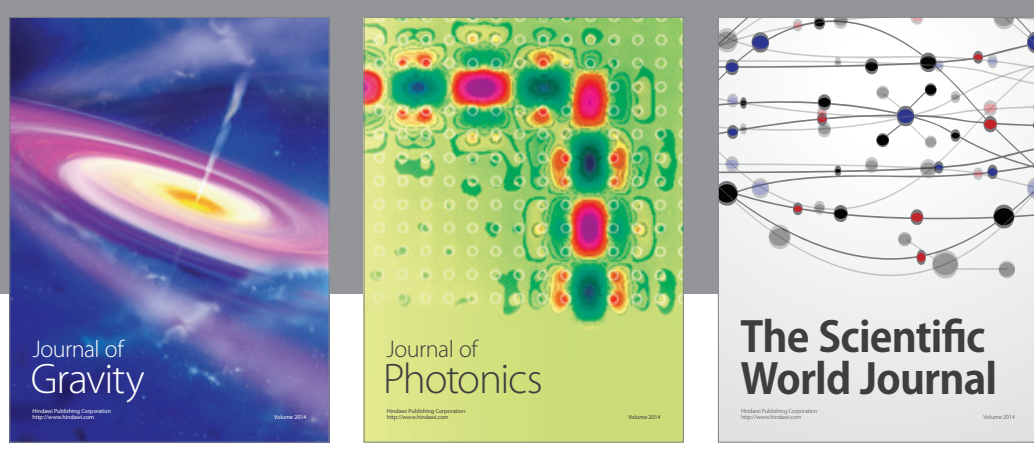

The Scientific World Journal
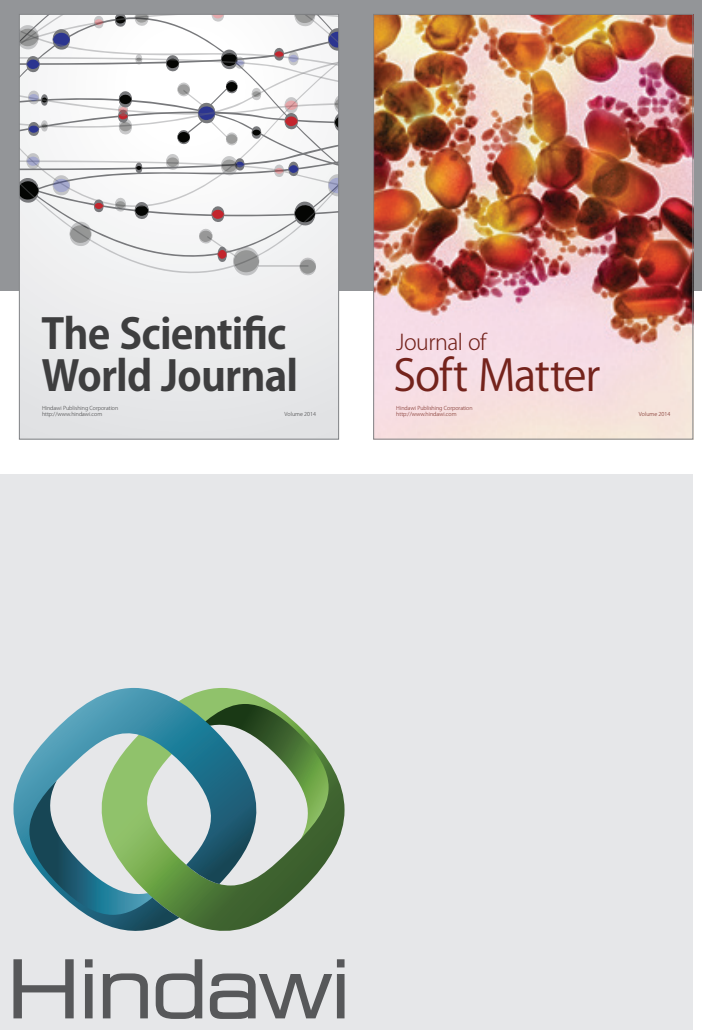

Submit your manuscripts at

https://www.hindawi.com
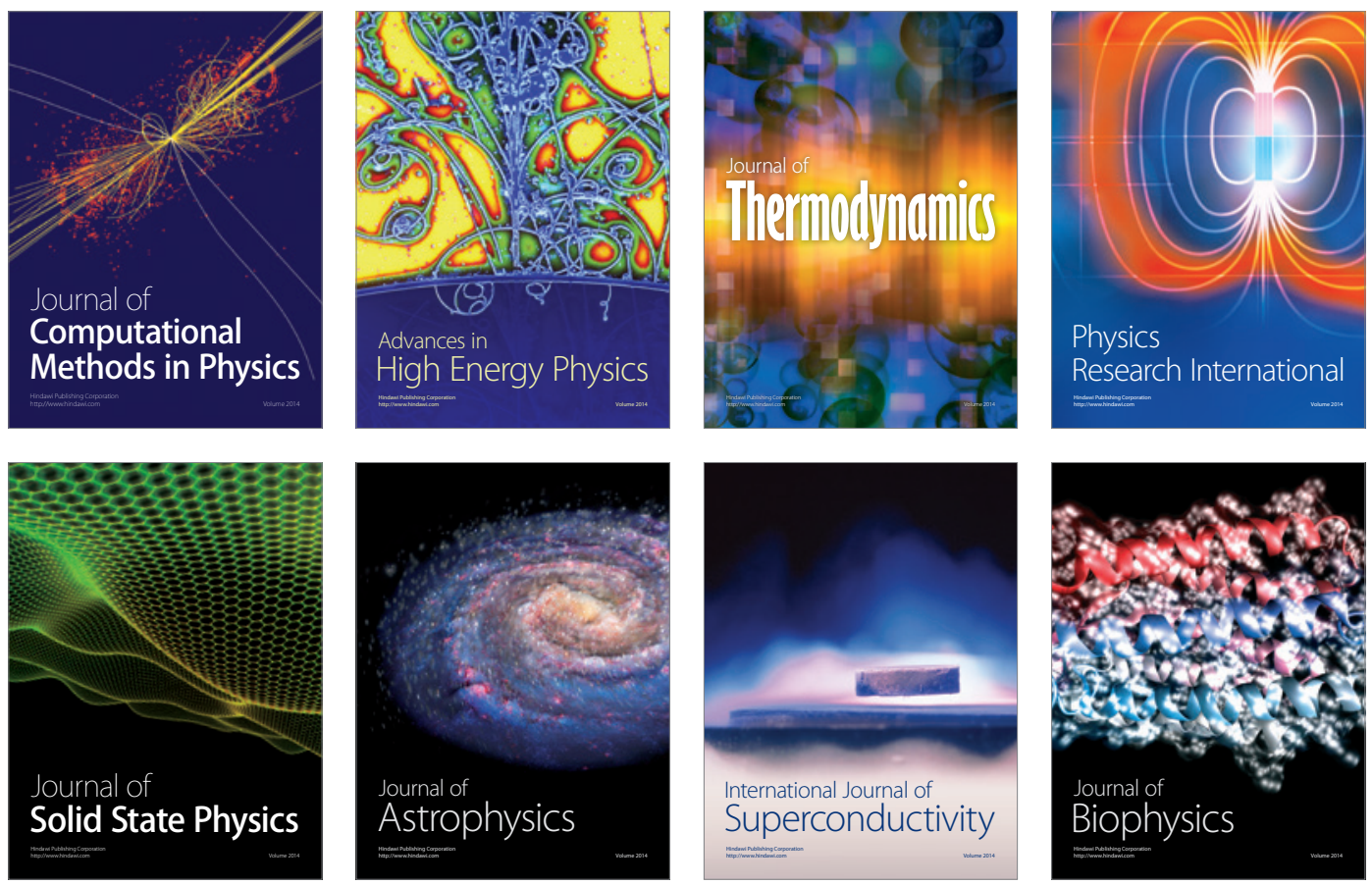
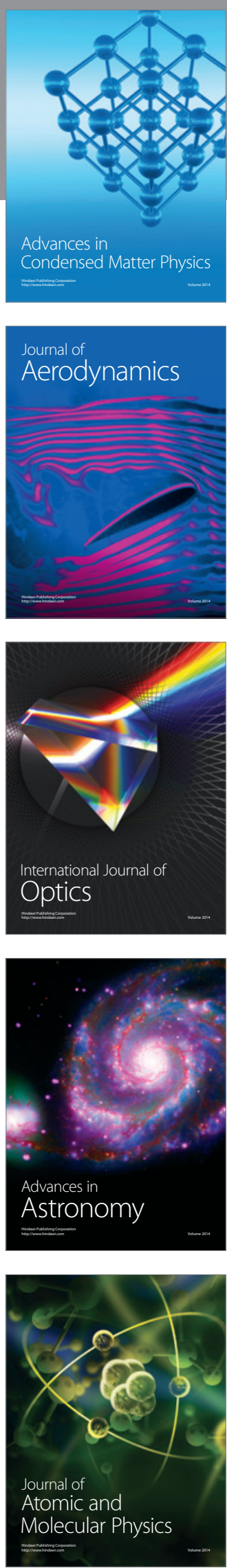